\title{
SOJOURN TIMES OF GAUSSIAN PROCESSES WITH TREND
}

\author{
KRZYSZTOF DȨBICKI, PENG LIU, AND ZBIGNIEW MICHNA
}

Abstract. We derive exact tail asymptotics of sojourn time above the level $u \geq 0$

$$
\mathbb{P}\left(v(u) \int_{0}^{T} \mathbb{I}(X(t)-c t>u) \mathrm{d} t>x\right), \quad x \geq 0
$$

as $u \rightarrow \infty$, where $X$ is a Gaussian process with continuous sample paths, $c$ is some constant, $v(u)$ is a positive function of $u$ and $T \in(0, \infty]$. Additionally, we analyze asymptotic distributional properties of

$$
\tau_{u}(x):=\inf \left\{t \geq 0: v(u) \int_{0}^{t} \mathbb{I}(X(s)-c s>u) \mathrm{d} s>x\right\},
$$

as $u \rightarrow \infty, x \geq 0$, where $\inf \emptyset=\infty$. The findings of this contribution are illustrated by a detailed analysis of a class of Gaussian processes with stationary increments and a family of self-similar Gaussian processes.

Key Words: cumulative Parisian ruin time; exact asymptotics; first passage time; Gaussian process with stationary increments; generalized Berman-type constant; self-similar Gaussian process; sojourn/occupation times.

AMS Classification: Primary 60G15; secondary 60G70

\section{INTRODUCTION}

Let $Y(t), t \in \mathbb{R}$ be a centered random process with càdlàg sample paths and let for $T>0$

$$
\int_{0}^{T} \mathbb{I}_{u}(Y(t)) \mathrm{d} t
$$

be the sojourn time of $Y$ above the level $u \in \mathbb{R}$ in interval $[0, T]$, where $\mathbb{I}_{u}(x)=\mathbb{I}(x>u)$. The asymptotic properties of (1), as $u \rightarrow \infty$ for $Y$ being a centered Gaussian process has been extensively studied by Berman, see e.g., [1-4]. An explicit form of the distribution of $(1)$ is known only for very few special processes. In particular, for $Y=B_{1}$ being a standard Brownian motion, by the arcsin law of Paul Lévy, we have

$$
\mathbb{P}\left(\int_{0}^{T} \mathbb{I}_{0}\left(B_{1}(t)\right) \mathrm{d} t>x\right)=1-\frac{2}{\pi} \arcsin \left(\sqrt{\frac{x}{T}}\right), \quad 0<x<T<\infty .
$$

An extension of this arcsin law is obtained in [5] for the case of $Y(t)=B_{1}(t)-c t, c \neq 0$. For the infinite time horizon, i.e., $T=\infty$ and $c>0$, in view of [6][Eq. (3), p. 255]

$$
\mathbb{P}\left(\int_{0}^{\infty} \mathbb{I}_{0}\left(B_{1}(t)-c t\right) \mathrm{d} t \in \mathrm{d} y\right)=\left(\frac{\sqrt{2} c}{\sqrt{\pi y}} e^{-\frac{c^{2} y}{2}}-\frac{2 c^{2}}{\sqrt{\pi}} \int_{\frac{c \sqrt{y}}{\sqrt{2}}}^{\infty} e^{-v^{2}} \mathrm{~d} v\right) \mathrm{d} y, \quad y \geq 0
$$

which implies that for any $x \geq 0$

$$
\mathbb{P}\left(\int_{0}^{\infty} \mathbb{I}_{0}\left(B_{1}(t)-c t\right) \mathrm{d} t>x\right)=2\left(1+c^{2} x\right) \Psi(c \sqrt{x})-\frac{c \sqrt{2 x}}{\sqrt{\pi}} e^{-\frac{c^{2} x}{2}},
$$

where $\Psi$ denotes the tail distribution of a standard normal random variable $N(0,1)$.

The above result can be extended to the class of spectrally negative Lévy processes with a negative drift and a general level $u$. Indeed, let $X$ be a spectrally negative Lévy process. Then for any non-negative $\lambda$

$$
\mathbb{E}\left\{e^{\lambda X(t)}\right\}=e^{t \psi(\lambda)}, \quad t \geq 0
$$

with $\psi$ being a strictly convex function such that $\lim _{\lambda \rightarrow \infty} \psi(\lambda)=\infty$ and $\psi^{\prime}(0+)=\mathbb{E}\{X(1)\}$.

Date: July 18, 2019. 
Theorem 1.1. Suppose that $X(t), t \geq 0$ is a spectrally negative Lévy process such that $\mathbb{E}\{X(1)\}<c$ for some $c>0$. Then for any $u \geq 0$

$$
\mathbb{P}\left(\int_{0}^{\infty} \mathbb{I}_{u}(X(t)-c t) \mathrm{d} t>x\right)=e^{-\alpha u} \mathbb{P}\left(\int_{0}^{\infty} \mathbb{I}_{0}(X(t)-c t) \mathrm{d} t>x\right),
$$

where $\alpha>0$ is the unique positive solution to $\psi(\alpha)=c \alpha$.

In the particular case that $X=B_{1}$, we have $\mathbb{E}\left\{B_{1}(1)\right\}=0<c$ and further $\psi(\alpha)=\frac{\alpha^{2}}{2}=c \alpha$ has the unique positive solution $\alpha=2 c$. Consequently, for any $x \geq 0$ and $u \in \mathbb{R}$ Theorem 1.1 combined with (3) implies

$$
\mathbb{P}\left(\int_{0}^{\infty} \mathbb{I}_{u}\left(B_{1}(t)-c t\right) \mathrm{d} t>x\right)=\left(2\left(1+c^{2} x\right) \Psi(c \sqrt{x})-\frac{c \sqrt{2 x}}{\sqrt{\pi}} e^{-\frac{c^{2} x}{2}}\right) e^{-2 c u}
$$

The study of distributional properties of occupation-type functionals for Lévy processes is crucial for many applications in finance and insurance (e.g. the occupation time in red or the inverse occupation time - the time of cumulative Parisian ruin), see for instance [5, 7-10]. The number of papers dealing with occupation times (sojourn times) is huge; most of the articles discuss the derivation of Laplace transform, see the recent contributions [11-14] and references therein. Recent paper [10] derives the density of occupation time for spectrally negative Lévy processes with exponential time horizon.

In this paper we shall focus on analogues of Theorem 1.1 for a wide class of Gaussian processes. Since the distribution of (1) is not tractable in the general Gaussian case, our investigation is concerned with the derivation of the exact asymptotic behavior of

$$
p_{T}(u, x):=\mathbb{P}\left(v(u) \int_{0}^{T} \mathbb{I}_{u}(X(t)-c t) \mathrm{d} t>x\right), \quad x \geq 0
$$

as $u \rightarrow \infty$, where $X$ is a Gaussian process with continuous sample paths, $v(u), u>0$ is a positive function (will be specified below) and $T \in(0, \infty]$. In order to avoid trivialities, we suppose that $c>0$ if $T=\infty$, while for $T<\infty$ we allow $c \in \mathbb{R}$. In contrast to the classical results for centered Gaussian processes $X$ by Berman, see e.g., [1-4], where the asymptotics of $\mathbb{P}\left(v(u) \int_{0}^{T} \mathbb{I}_{u}(X(t)) \mathrm{d} t>x\right)$ as $u \rightarrow \infty$ is given for a.e. $x \geq 0$, an important advantage of the technique used in this contribution allowed us to show that the asymptotics of (6) holds for all $x \geq 0$. This is due to continuity of Berman-type constant which shall be proven in Lemma 4.1.

Additionally, motivated by recent investigations on the ruin time, see e.g., [15] and [16], we shall analyze asymptotic distributional properties of

$$
\tau_{u}(x):=\inf \left\{t \geq 0: v(u) \int_{0}^{t} \mathbb{I}_{u}(X(s)-c s) \mathrm{d} s>x\right\}
$$

as $u \rightarrow \infty, x \geq 0$, with the convention that $\inf \emptyset=\infty$. Note that $\tau_{u}(x)$ is called the cumulative Parisian ruin time in [9], which is of interest in risk theory; $\tau_{u}(0)$ is the first passage time of the level $u$ by the process $X(t)-c t$, which is referred to as the ruin time in [15]. In Section 3 a distributional approximation, as $u \rightarrow \infty$, of $\tau_{u}^{*}\left(x_{1}, x_{2}\right)$ defined by

$$
\tau_{u}^{*}\left(x_{1}, x_{2}\right):=\tau_{u}\left(x_{2}\right) \mid \tau_{u}\left(x_{1}\right)<\infty, \quad 0 \leq x_{1} \leq x_{2}<\infty
$$

is derived.

Brief outline of the paper: In Section 2 we derive an exact approximation of sojourn times for general Gaussian processes. In Section 3 we apply this result to (6), for $X$ being a centered Gaussian process with stationary increments and a self-similar Gaussian process. Both scenarios $T \in(0, \infty)$ and $T=\infty$ are considered. Section 4 contains some lemmas that are useful in the proofs of the main results, while the proofs are presented in Section 5. 


\section{MAIN RESULT}

In this section we provide a general result preparing us to derive exact asymptotics of (6) for a wide class of centered Gaussian process $X(t), t \in \mathbb{R}$ with continuous trajectories. In order to motivate the study of this section write first for $u>0, x \geq 0, c>0$ and $v(u)$ representing an arbitrary positive function

$$
\begin{aligned}
p_{\infty}(u, x) & :=\mathbb{P}\left(v(u) \int_{0}^{\infty} \mathbb{I}_{u}(X(t)-c t) \mathrm{d} t>x\right) \\
& =\mathbb{P}\left(v(u) u \int_{0}^{\infty} \mathbb{I}_{0}(X(u t)-c u t-u) \mathrm{d} t>x\right) \\
& =\mathbb{P}\left(v(u) u \int_{0}^{\infty} \mathbb{I}_{M(u)}\left(\frac{X(u t)}{u(1+c t)} M(u)\right) \mathrm{d} t>x\right)
\end{aligned}
$$

where $M(u)=\inf _{t \in[0, \infty)} \frac{u(1+c t)}{\sqrt{\operatorname{Var}(X(u t))}}$ is assumed to be positive. Then $\frac{X(u t)}{u(1+c t)} M(u)$ is a Gaussian process with mean 0 and $\sup _{t \geq 0} \operatorname{Var}\left(\frac{X(u t)}{u(1+c t)} M(u)\right)=1$. As it will be proven in Section 5 , for $\delta_{u}$, a properly chosen function of $u$ with $\lim _{u \rightarrow \infty} \delta_{u}=0$, and $t_{u}=\arg \inf _{t \geq 0} \frac{u(1+c t)}{\sqrt{\operatorname{Var}(X(u t))}}$ for $x \geq 0$ we have

$$
\begin{aligned}
p_{\infty}(u, x) & \sim \mathbb{P}\left(v(u) u \int_{\left[t_{u}-\delta_{u}, t_{u}+\delta_{u}\right]} \mathbb{I}_{M(u)}\left(\frac{X(u t)}{u(1+c t)} M(u)\right) \mathrm{d} t>x\right) \\
& =\mathbb{P}\left(\int_{\left[-v(u) u \delta_{u}, v(u) u \delta_{u}\right]} \mathbb{I}_{M(u)}\left(\frac{X\left(u t_{u}+t / v(u)\right)}{u\left(1+c t_{u}\right)+c t / v(u)} M(u)\right) \mathrm{d} t>x\right) .
\end{aligned}
$$

A similar transformation for $p_{T}(u, x), T \in(0, \infty)$ shows that in general the problem to deal with can be reduced to

$$
p_{T}(u, x) \sim \mathbb{P}\left(\int_{E(u)} \mathbb{I}_{n(u)}\left(Z_{u}(t)\right) \mathrm{d} t>x\right), \quad u \rightarrow \infty,
$$

where $x \geq 0, n(u)$ is a function of $u$ and $Z_{u}, u>0$ is a family of centered Gaussian processes with continuous trajectories defined on the interval $E(u)=\left[a_{1}(u), a_{2}(u)\right]$.

In the rest of this paper we shall impose some standard assumptions on the behaviour of the variance function $\sigma_{u}$ and the correlation function $r_{u}$ of $Z_{u}$. In particular, we shall assume that

$$
\lim _{u \rightarrow \infty} \sup _{t \neq 0, t \in E(u)}\left|\frac{1-\sigma_{u}(t)}{w(g(u)|t|)}-1\right|=0
$$

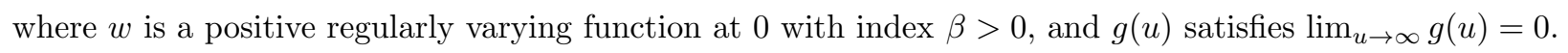

In the following $\Delta(u), n(u), u>0$ are positive functions such that

$$
\lim _{u \rightarrow \infty} \Delta(u)=\varphi \in[0, \infty], \quad \lim _{u \rightarrow \infty} n(u)=\infty
$$

For the correlation function of $Z_{u}$ we shall assume that for $\Delta(u)$ satisfying (12) we have

$$
\lim _{u \rightarrow \infty} \sup _{s \neq t, s, t \in E(u)}\left|\frac{n^{2}(u)\left(1-r_{u}(s, t)\right)}{\frac{\sigma_{\eta}^{2}(\Delta(u)|t-s|)}{\sigma_{\eta}^{2}(\Delta(u))}-1}\right|=0,
$$

where $\eta(t), t \in \mathbb{R}$ is a centered Gaussian process with continuous trajectories, stationary increments and variance function $\sigma_{\eta}^{2}(t)>0, t>0$, being regularly varying at 0 and at $\infty$ with indexes $2 \alpha_{0} \in(0,2]$ and $2 \alpha_{\infty} \in(0,2)$, respectively.

Assumptions (11) and (13) are satisfied for large classes of Gaussian processes, see e.g., [17], [18], [19] and [20]. For example, they are compatible with those in Theorem 3.2 in [21].

Next, for any $\varphi \in[0, \infty]$ set

$$
\eta_{\varphi}(t)=\left\{\begin{array}{cc}
B_{2 \alpha_{0}}(t), & \varphi=0 \\
\frac{\eta(\varphi t)}{\sigma_{\eta}(\varphi)}, & \varphi \in(0, \infty) \\
B_{2 \alpha_{\infty}}(t), & \varphi=\infty,
\end{array}\right.
$$


where $B_{\alpha}$ is a fractional Brownian motion (fBm) with self-similarity index $\alpha / 2 \in(0,1]$ and process $\eta$ is defined above. For a random process $W(t), t \in \mathbb{R}$ with continuous trajectories, $x \geq 0, E$ a compact subset of $\mathbb{R}$ and $h$ a continuous function on $E$ we define

$$
\mathcal{B}_{W}^{h}(x, E)=\int_{\mathbb{R}} \mathbb{P}\left(\int_{E} \mathbb{I}_{0}(\sqrt{2} W(t)-\operatorname{Var}(W(t))-h(t)+z) \mathrm{d} t>x\right) e^{-z} \mathrm{~d} z
$$

and when the limit exists, we set

$$
\mathcal{B}_{W}^{h}(x)=\lim _{S \rightarrow \infty} \frac{\mathcal{B}_{W}^{h}(x,[0, S])}{S^{\llbracket(h=0)}} .
$$

Moreover, set below

$$
\widehat{\mathcal{B}}_{B_{\alpha}}^{h}(x)=\lim _{S \rightarrow \infty} \mathcal{B}_{B_{\alpha}}^{h}(x,[-S, S]),
$$

provided that the above limit is finite. For $h=0$, we suppress the superscript and write $\mathcal{B}_{W}(x)$ or $\mathcal{B}_{W}(x, E)$. If $W=B_{\alpha}$ with $B_{\alpha}$ fractional Brownian motion, then $\mathcal{B}_{B_{\alpha}}(0)$ is simply the Pickands constant, see e.g., [22], [18], [19] and $[20]$.

If $h$ is strictly positive, then $\mathcal{B}_{B_{\alpha}}^{h}(0)$ and $\widehat{\mathcal{B}}_{B_{\alpha}}^{h}(0)$ reduce to Piterbarg constants. We refer to [17-20] for the existence and properties of Pickands and Piterbarg related constants. All our asymptotic results below hold for all $x \geq 0$, which generalize the results in [1]-[4] and [23], where the asymptotics hold for almost all $x \in[0, \infty)$.

Throughout this paper, $\overleftarrow{f}$ stands for the generalized asymptotic (unique) inverse of a regularly varying function $f$ see [24].

Next we present the main result of this contribution.

Theorem 2.1. Let $Z_{u}(t), t \in E(u)$ with $E(u):=\left[a_{1}(u), a_{2}(u)\right]$ be a family of centered Gaussian processes with continuous trajectories. Suppose that (11)-(13) hold, $\theta(u)=\overleftarrow{w}\left(n^{-2}(u)\right) / g(u)$ and

$$
\lim _{u \rightarrow \infty} n^{2}(u) w(g(u))=\gamma \in[0, \infty], \quad \lim _{u \rightarrow \infty} g(u)\left|a_{i}(u)\right|=0, i=1,2 .
$$

i) If $\gamma=0$ and

$$
\lim _{u \rightarrow \infty} n^{2}(u) w\left(g(u)\left|a_{i}(u)\right|\right)=x_{i}, \quad \lim _{u \rightarrow \infty} n(u) w\left(g(u)\left|a_{i}(u)\right|\right)=0, \quad i=1,2,
$$

then

$$
\mathbb{P}\left(\int_{E(u)} \mathbb{I}_{n(u)}\left(Z_{u}(t)\right) \mathrm{d} t>x\right) \sim \mathcal{B}_{\eta_{\varphi}}(x) \frac{1}{\beta} \int_{y_{1}}^{y_{2}}|t|^{1 / \beta-1} e^{-|t|} \mathrm{d} t \theta(u) \Psi(n(u)),
$$

with $y_{2}-y_{1}>0$ and

$$
\left.y_{i}=x_{i} \mathbb{I}\left(x_{i}>0, \lim _{u \rightarrow \infty} a_{i}(u)=\infty\right\}\right)-x_{i} \mathbb{I}\left(x_{i}>0, \lim _{u \rightarrow \infty} a_{i}(u)=-\infty\right), \quad i=1,2 .
$$

ii) If $\gamma \in(0, \infty)$ and $\lim _{u \rightarrow \infty} a_{i}(u)=a_{i}, i=1,2$, with $a_{1} \in[-\infty, 0], a_{2} \in[0, \infty]$ and $a_{2}-a_{1}>x$, then

$$
\mathbb{P}\left(\int_{E(u)} \mathbb{I}_{n(u)}\left(Z_{u}(t)\right) \mathrm{d} t>x\right) \sim \mathcal{B}_{\eta_{\varphi}}^{\gamma|t|^{\beta}}\left(x,\left[a_{1}, a_{2}\right]\right) \Psi(n(u)) .
$$

iii) If $\gamma=\infty$ and

$$
\lim _{u \rightarrow \infty} \frac{a_{i}(u)}{\theta(u)}=b_{i}, \quad i=1,2,
$$

with $b_{1} \in[-\infty, \infty), b_{2} \in(-\infty, \infty]$, and $b_{2}-b_{1}>x$, then

$$
\mathbb{P}\left(\int_{E(u)} \mathbb{I}_{n(u)}\left(Z_{u}(t)\right) \mathrm{d} t>\theta(u) x\right) \sim \mathcal{B}_{0}^{|t|^{\beta}}\left(x,\left[b_{1}, b_{2}\right]\right) \Psi(n(u)) .
$$

Remark 2.2. i) If we assume that $\lim _{u \rightarrow \infty} \Delta(u)=0$ and $\sigma_{\eta}^{2}$ in (13) is a non-negative regularly varying function at 0 with index $2 \alpha_{0} \in(0,2]$, then Theorem 2.1 still hold with $\eta_{\varphi}$ replaced by $B_{2 \alpha_{0}}$.

ii) The case $x=0$ in Theorem 2.1 generalizes the results in [17], [18], [19] and [20] and together with i) of this remark covers the results for one dimensional case in [22].

In the following lemma we calculate the exact value of two special Berman constants. 
Lemma 2.3. For $\gamma, \beta>0$

$$
\mathcal{B}_{0}^{\gamma t^{\beta}}(x)=\mathcal{B}_{0}^{\gamma t^{\beta}}(x,[0, \infty))=e^{-\gamma x^{\beta}}, \quad \mathcal{B}_{0}^{\gamma|t|^{\beta}}(x,(-\infty, y])=\left\{\begin{array}{cc}
e^{-\gamma(x-y)^{\beta}}, & y<x / 2 \\
e^{-\gamma 2^{-\beta} x^{\beta}} & y \geq x / 2
\end{array}\right.
$$

and $\mathcal{B}_{0} \gamma|t|^{\beta}\left(x,\left[b_{1}, b_{2}\right]\right)$ is continuous for all $x \geq 0$. For any $x \geq 0$ and $\gamma>0$

$$
\widehat{\mathcal{B}}_{B_{2}}^{\gamma t^{2}}(x)=\sqrt{\frac{1+\gamma}{\gamma}} e^{-\frac{(1+\gamma) x^{2}}{4}} .
$$

\section{Applications}

In this section we apply Theorem 2.1 to two classes of Gaussian processes: Gaussian processes with stationary increments and self-similar Gaussian processes.

3.1. Gaussian processes with stationary increments. Given $X(t), t \in \mathbb{R}$ a Gaussian process with stationary increments and continuous sample paths, we consider

$$
p_{T}(u, x):=\mathbb{P}\left(v(u) \int_{0}^{T} \mathbb{I}_{u}(X(t)-c t) \mathrm{d} t>x\right), \quad x \geq 0
$$

where the positive scaling function $v$ will be specified later, and $T \in(0, \infty]$. Let $\sigma^{2}(t)=\operatorname{Var}(X(t))$. We distinguish two cases, leading to qualitatively different asymptotics, namely $T \in(0, \infty)$ and $T=\infty$.

3.1.1. Infinite time horizon. By the stationarity of increments of $X$ the covariance function of $X$ is completely determined by its variance function $\sigma^{2}$. Along the same lines as in [20] or [25], we assume that

AI: $\sigma^{2}(0)=0$ and $\sigma^{2}(t)$ is regularly varying at $\infty$ with index $2 \alpha_{\infty} \in(0,2)$. Further, $\sigma^{2}(t)$ is twice continuously differentiable on $(0, \infty)$ with its first derivative $\dot{\sigma}^{2}(t):=\frac{\mathrm{d} \sigma^{2}}{\mathrm{~d} t}(t)$ and second derivative $\ddot{\sigma^{2}}(t):=\frac{\mathrm{d}^{2} \sigma^{2}}{\mathrm{~d} t^{2}}(t)$ being ultimately monotone at $\infty$.

AII: $\sigma^{2}(t)$ is regularly varying at 0 with index $2 \alpha_{0} \in(0,2]$.

Assumptions AI-AII cover a wide range of Gaussian processes with stationary increments, including two important families: (1) fractional Brownian motions $B_{\alpha}(t), \alpha \in(0,2]$ and $(2)$ Gaussian integrated processes, i.e., the case where $X(t)=\int_{0}^{t} Z(s) \mathrm{d} s$, with $Z$ a centered continuous stationary Gaussian process with variance 1 and correlation function $r(s)=\operatorname{Cov}(X(t), X(t+s)), s, t \geq 0$ satisfying some regularity conditions; see, e.g., [18], [19], [20], [25] and $[26]$.

Suppose that $c>0$ and let in the following $(\overleftarrow{\sigma}$ stands for the asymptotic inverse of $\sigma)$

$$
1 / v(u)=\overleftarrow{\sigma}\left(\frac{\sqrt{2} \sigma^{2}\left(u t^{*}\right)}{u\left(1+c t^{*}\right)}\right), \quad t^{*}=\frac{\alpha_{\infty}}{c\left(1-\alpha_{\infty}\right)}
$$

According to (9), recall that

$$
p_{\infty}(u, x)=\mathbb{P}\left(u v(u) \int_{0}^{\infty} \mathbb{I}_{M(u)}\left(\frac{X(u t)}{u(1+c t)} M(u)\right) \mathrm{d} t>x\right),
$$

where

We note that

$$
M(u)=\inf _{t>0} \operatorname{Var}^{-1 / 2}\left(\frac{X(u t)}{u(1+c t)}\right)=\inf _{t>0} \frac{u(1+c t)}{\sigma(u t)} .
$$

$$
\frac{X(u t)}{u(1+c t)} M(u), \quad t \geq 0
$$

is a family of centered Gaussian processes with the maximum of their variance functions equal to 1.

Applying Theorem 2.1 we arrive at the following results, where

$$
A=\left(\frac{\alpha_{\infty}}{c\left(1-\alpha_{\infty}\right)}\right)^{-\alpha_{\infty}} \frac{1}{1-\alpha_{\infty}}, \quad B=\left(\frac{\alpha_{\infty}}{c\left(1-\alpha_{\infty}\right)}\right)^{-\alpha_{\infty}-2} \alpha_{\infty}
$$


Theorem 3.1. Let $X(t), t \in \mathbb{R}$ be a centered Gaussian process with continuous trajectories and stationary increments satisfying AI-AII and $c>0$. If

$$
\varphi=\lim _{u \rightarrow \infty} \frac{\sigma^{2}(u)}{u} \in[0, \infty]
$$

then for any $x \geq 0$

$$
p_{\infty}(u, x) \sim \mathcal{B}_{X_{\varphi}}(x) \sqrt{\frac{2 A \pi}{B}} \frac{u}{M(u) \overleftarrow{\sigma}\left(\frac{\sqrt{2} \sigma^{2}\left(u t^{*}\right)}{u\left(1+c t^{*}\right)}\right.} \Psi(M(u))
$$

where

$$
X_{\varphi}(t)= \begin{cases}B_{2 \alpha_{0}}(t), & \text { if } \varphi=0 \\ \frac{1+c t^{*}}{\sqrt{2} \varphi t^{*}} X\left(\overleftarrow{\sigma}\left(\frac{\sqrt{2} \varphi t^{*}}{1+c t^{*}}\right) t\right), & \text { if } \varphi \in(0, \infty) \\ B_{2 \alpha_{\infty}}(t), & \text { if } \varphi=\infty\end{cases}
$$

Application of Theorem 3.1 to $X=B_{1}$ with comparison to (5) leads to the following corollary.

Corollary 3.2. For any $x \geq 0$,

$$
\mathcal{B}_{B_{1}}(x)=(2+x) \Psi\left(\sqrt{\frac{x}{2}}\right)-\sqrt{\frac{x}{\pi}} e^{-\frac{x}{4}}
$$

Next we analyze the asymptotic distribution of $\tau_{u}^{*}\left(x_{1}, x_{2}\right)$ defined in (8), assuming that these random variables are defined on the same probability space.

Corollary 3.3. Under the assumptions of Theorem 3.1, for $0 \leq x_{1} \leq x_{2}<\infty$, the following convergence in distribution holds

$$
\frac{\tau_{u}^{*}\left(x_{1}, x_{2}\right)-u t_{u}}{A(u)} \stackrel{d}{\rightarrow} \mathcal{N}_{x_{1}, x_{2}}, \quad u \rightarrow \infty
$$

where

$$
\mathbb{P}\left(\mathcal{N}_{x_{1}, x_{2}} \leq y\right)=\frac{\mathcal{B}_{X_{\varphi}}\left(x_{2}\right)}{\mathcal{B}_{X_{\varphi}}\left(x_{1}\right)} \mathbb{P}(\mathcal{N} \leq y), \quad y \in \mathbb{R}, \quad \mathbb{P}\left(\mathcal{N}_{x_{1}, x_{2}}=\infty\right)=1-\frac{\mathcal{B}_{X_{\varphi}}\left(x_{2}\right)}{\mathcal{B}_{X_{\varphi}}\left(x_{1}\right)},
$$

with $A(u)=\frac{\sigma\left(u t^{*}\right)}{c} \sqrt{\frac{\alpha_{\infty}}{1-\alpha_{\infty}}}$ and $\mathcal{N}$ an $N(0,1)$ random variable.

3.1.2. Finite time horizon. In this subsection we consider the finite-time horizon case, i.e., we are interested in the asymptotics of $p_{T}(u, x)$ as $u \rightarrow \infty$, where $X$ has stationary increments, $x \geq 0, T \in(0, \infty)$ and $c \in \mathbb{R}$. Due to the finiteness of $T$, we allow in this section $c \in \mathbb{R}$. Clearly,

$$
\begin{aligned}
p_{T}(u, x) & =\mathbb{P}\left(v(u) \int_{0}^{T} \mathbb{I}_{0}\left(\frac{X(t)}{u+c t}-1\right) \mathrm{d} t>x\right) \\
& =\mathbb{P}\left(v(u) \int_{0}^{T} \mathbb{I}_{m(u)}\left(\frac{X(t)}{u+c t} m(u)\right) \mathrm{d} t>x\right)
\end{aligned}
$$

where

$$
m(u)=\frac{u+c T}{\sigma(T)} .
$$

We shall impose the following assumptions on $\sigma$.

BI $\sigma(0)=0$ and $\sigma \in C([0, T])$ with the first derivative $\dot{\sigma}(t)>0, t \in(0, T]$.

BII $\sigma$ is regularly varying at 0 with index $\alpha_{0} \in(0,1]$.

We note that both $\mathrm{fBm}$ and introduced in Section 3.1.1 Gaussian integrated processes $\int_{0}^{t} Z(s) \mathrm{d} s$ with correlation of $Z$ such that $r(t)>0$ satisfy conditions BI-BII (note that $\sigma^{2}(t)=2 \int_{0}^{t} \int_{0}^{s} r(u) \mathrm{d} u \mathrm{~d} s, t \geq 0$ and $\alpha_{0}=1$ ).

Assumption BI ensures that the first derivative of $\frac{\sigma(t)}{u+c t}$ is positive and further its maximizer over $[0, T]$ is unique and equals $T$ for sufficiently large $\mathrm{u}$. Assumption BII gives the correlation structure of $\frac{X(t)}{u+c t}$ around time $t=T$ (see Lemma $4.4 \mathrm{ii})$. Thus under the assumptions of BI-BII, $\frac{X(t)}{u+c t} m(u), t \in[0, T]$ is a centered continuous Gaussian process 
with the maximum of variance function attained at $t=T$ and equal to 1 .

Set

$$
v(u)=\left\{\begin{array}{cc}
1 / \overleftarrow{\sigma}\left(\frac{\sqrt{2} \sigma^{2}(T)}{u+c T}\right), & \lim _{t \rightarrow 0} \frac{|t|}{\sigma^{2}(|t|)} \in[0, \infty) \\
(m(u))^{2}, & \lim _{t \rightarrow 0} \frac{|t|}{\sigma^{2}(|t|)}=\infty
\end{array}\right.
$$

Theorem 3.4. Suppose that $X(t), t \in[0, T]$ is a centered continuous Gaussian process with stationary increments satisfying BI-BII and let $x \geq 0, c \in \mathbb{R}$ be given.

i) If $t=o\left(\sigma^{2}(t)\right)$ as $t \rightarrow 0$, then

$$
p_{T}(u, x) \sim \mathcal{B}_{B_{2 \alpha_{0}}}(x) \frac{\sigma(T)}{\dot{\sigma}(T)} \frac{1}{(m(u))^{2} \overleftarrow{\sigma}\left(\frac{\sqrt{2} \sigma^{2}(T)}{u+c T}\right)} \Psi(m(u))
$$

ii) If $\lim _{t \rightarrow 0} \sigma^{2}(t) / t=\theta \in(0, \infty)$, then

$$
p_{T}(u, x) \sim \mathcal{B}_{B_{1}}^{\frac{2 \sigma(T) \dot{\sigma}(T)}{\theta}|t|}(x) \Psi(m(u)) .
$$

iii) If $\sigma^{2}(t)=o(t)$ as $t \rightarrow 0$, then

$$
p_{T}(u, x) \sim e^{-\frac{\dot{\sigma}(T)}{\sigma(T)} x} \Psi(m(u))
$$

With the convention that $\inf \emptyset=\infty$, define $\tau_{u, T}(x), T>0, x \geq 0$ by

$$
\tau_{u, T}(x)=\inf \left\{t: v(u) \int_{0}^{t} \mathbb{I}_{u}(X(s)-c s) \mathrm{d} s>x, 0 \leq t \leq T\right\} .
$$

Further, let

$$
\tau_{u, T}^{*}\left(x_{1}, x_{2}\right):=\tau_{u, T}\left(x_{2}\right) \mid \tau_{u, T}\left(x_{1}\right) \leq T, \quad 0 \leq x_{1} \leq x_{2}<\infty
$$

Corollary 3.5. Suppose that $X(t), t \in[0, T]$ is a centered continuous Gaussian process with stationary increments satisfying BI-BII, $0 \leq x_{1} \leq x_{2}<\infty, c \in \mathbb{R}$ are given and

$$
\lim _{t \rightarrow 0} \frac{|t|}{\sigma^{2}(|t|)} \in[0, \infty]
$$

Then

$$
\frac{\dot{\sigma}(T)}{\sigma^{3}(T)} u^{2}\left(T-\tau_{u, T}^{*}\left(x_{1}, x_{2}\right)\right) \stackrel{d}{\rightarrow} \mathcal{E}_{x_{1}, x_{2}}, \quad 0 \leq x_{1} \leq x_{2}<\infty, \quad u \rightarrow \infty
$$

where

$$
\mathbb{P}\left(\mathcal{E}_{x_{1}, x_{2}}>y\right)=\Gamma\left(x_{1}, x_{2}\right) e^{-y}, \quad \mathbb{P}\left(\mathcal{E}_{x_{1}, x_{2}}=-\infty\right)=1-\Gamma\left(x_{1}, x_{2}\right) \geq 0, \quad y \geq 0
$$

with

$$
\Gamma\left(x_{1}, x_{2}\right)=\left\{\begin{array}{cl}
\frac{\mathcal{B}_{B_{2 \alpha_{0}}}\left(x_{2}\right)}{\overline{\mathcal{B}}_{B_{2} \alpha_{0}}\left(x_{1}\right)} & t=o\left(\sigma^{2}(t)\right) \\
\frac{\mathcal{B}_{B_{1}}^{\frac{2 \sigma(T) \dot{\sigma}(T)}{\theta}|t|}\left(x_{2}\right)}{\mathcal{B}_{B_{1}}^{\frac{2 \sigma(T) \dot{\sigma}(T)}{\theta}|t|}\left(x_{1}\right)} & \sigma^{2}(t) \sim \theta t \quad, \quad 0 \leq x_{1} \leq x_{2}<\infty . \\
e^{\frac{\sigma(T)}{\sigma(T)}\left(x_{1}-x_{2}\right)} & \sigma^{2}(t)=o(t)
\end{array}\right.
$$

3.2. Self-similar Gaussian processes. In this subsection we apply our findings to the class of self-similar Gaussian processes with drift. We focus on the exact asymptotic probabilities of sojourn time, without specifying analogs of Corollaries 3.3 and 3.5, since they essentially lead to the same type of results as given in Section 3.1.

Suppose that $X$ is a centered self-similar Gaussian process with self-similarity index $H \in(0,1)$, i.e.

$$
\{X(b t), t \geq 0\} \stackrel{d}{=}\left\{b^{H} X(t), t \geq 0\right\}
$$

where $\stackrel{d}{=}$ means the equality of finite dimensional distributions.

Equality (29) implies that $\sigma^{2}(t)=\operatorname{Var}(X(t))=\operatorname{Var}(X(1)) t^{2 H}$. Without loss of generality, in what follows we assume that $\operatorname{Var}(X(1))=1$ and hence $\sigma^{2}(t)=t^{2 H}$.

Moreover, we assume that 
$\mathbf{S}$ There exist a function $\rho$ which is regularly varying at 0 with index $\alpha \in(0,2], \rho(0)=0$ and $\rho(t)>0, t>0$, and $t_{0} \in[0, T]$ such that

$$
\lim _{\epsilon \rightarrow 0} \sup _{s \neq t,\left|s-t_{0}\right|<\epsilon,\left|t-t_{0}\right|<\epsilon, s, t \in[0, T]}\left|\frac{1-\operatorname{Corr}(X(s), X(t))}{\rho(|t-s|)}-1\right|=0 .
$$

Condition $\mathbf{S}$ is satisfied by such classes of self-similar Gaussian processes as fBms, bi-fractional Brownian motions, sub-fractional Brownian motions or generalized fractional Brownian motions; see, e.g., [27], [28], [29] or [30].

3.2.1. Infinite-time horizon. Suppose that $T=\infty$ and $c>0$. Then, by self-similarity of $X$, we have

$$
\mathbb{P}\left(v(u) \int_{0}^{\infty} \mathbb{I}_{u}(X(t)-c t) d t>x\right)=\mathbb{P}\left(u v(u) \int_{0}^{\infty} \mathbb{I}_{u^{1-H}}\left(\frac{X(t)}{1+c t}\right) d t>x\right) .
$$

Note that the maximizer of $\sqrt{\operatorname{Var}\left(\frac{X(t)}{1+c t}\right)}=\frac{t^{H}}{1+c t}$ is unique and equals $\frac{H}{c(1-H)}$. Further, referring to [17],

$$
\widehat{A} \frac{t^{H}}{1+c t}=1-\frac{\widehat{B}}{2 \widehat{A}}\left(t-\frac{H}{c(1-H)}\right)^{2}(1+o(1)), \quad t \rightarrow \frac{H}{c(1-H)}
$$

with

We arrive at the following result.

$$
\widehat{A}=\left(\frac{H}{c(1-H)}\right)^{-H} \frac{1}{1-H}, \quad \widehat{B}=\left(\frac{H}{c(1-H)}\right)^{-H-2} H
$$

Theorem 3.6. Let $X(t)$ be a centered self-similar Gaussian process with self-similarity index $H \in(0,1)$ satisfying (29) and $\mathbf{S}$ with $t_{0}=\frac{H}{c(1-H)}$. Suppose that

$$
\lim _{t \rightarrow 0} \frac{t^{2}}{\rho(t)}=\gamma \in[0, \infty]
$$

and let $x \geq 0, c>0$ be given.

i) If $\gamma=0$, then

$$
\mathbb{P}\left(\frac{1}{u \overleftarrow{\rho}\left(\left(\widehat{A} u^{1-H}\right)^{-2}\right)} \int_{0}^{\infty} \mathbb{I}_{u}(X(t)-c t) d t>x\right) \sim \mathcal{B}_{B_{\alpha}}(x) \sqrt{\frac{2 \widehat{A} \pi}{\widehat{B}}} \frac{1}{\overleftarrow{\rho}\left(\left(\widehat{A} u^{1-H}\right)^{-2}\right) \widehat{A} u^{1-H}} \Psi\left(\widehat{A} u^{1-H}\right)
$$

ii) If $\gamma \in(0, \infty)$, then

$$
\mathbb{P}\left(\frac{1}{u \overleftarrow{\rho}\left(\left(\widehat{A} u^{1-H}\right)^{-2}\right)} \int_{0}^{\infty} \mathbb{I}_{u}(X(t)-c t) d t>x\right) \sim \sqrt{\frac{2 \widehat{A}+\widehat{B} \gamma}{\widehat{B} \gamma}} e^{-\frac{2 \widehat{A}+\widehat{B} \gamma}{8 \widehat{A}} x^{2}} \Psi\left(\widehat{A} u^{1-H}\right)
$$

iii) If $\gamma=\infty$, then

$$
\mathbb{P}\left(u^{-H} \int_{0}^{\infty} \mathbb{I}_{u}(X(t)-c t) d t>x\right) \sim e^{-\frac{\widehat{A} \widehat{B} x^{2}}{8}} \Psi\left(\widehat{A} u^{1-H}\right) .
$$

3.2.2. Finite-time horizon. Let $T \in(0, \infty)$. We arrive at the following result.

Theorem 3.7. Let $X(t)$ be a centered self-similar Gaussian process with self-similarity index $H \in(0,1)$ satisfying (29) and $\mathbf{S}$ with $t_{0}=T$. For given $x \geq 0$ and $c \in \mathbb{R}$, let $p_{T}(u, x)$ be defined in (21) with $v(u)=\overleftarrow{\rho}\left(\frac{T^{2 H}}{(u+c T)^{2}}\right)$ if $\lim _{t \rightarrow 0} \frac{|t|}{\rho(|t|)} \in[0, \infty)$ and $v(u)=\frac{(u+c T)^{2}}{T^{2 H}}$ if $\lim _{t \rightarrow 0} \frac{|t|}{\rho(|t|)}=\infty$.

i) If $t=o(\rho(t))$ as $t \rightarrow 0$, then

$$
p_{T}(u, x) \sim \mathcal{B}_{B_{\alpha}}(x) \frac{T^{2 H+1-2 H / \alpha}}{H} \frac{1}{u^{2} \overleftarrow{\rho}\left(u^{-2}\right)} \Psi\left(\frac{u+c T}{T^{H}}\right) .
$$

ii) If $\lim _{t \rightarrow 0} \rho(t) / t=\theta$, then

$$
p_{T}(u, x) \sim \mathcal{B}_{B_{1}}^{\frac{H}{T \theta}|t|}(x) \Psi\left(\frac{u+c T}{T^{H}}\right) .
$$

iii) If $\rho(t)=o(t)$ as $t \rightarrow 0$, then

$$
p_{T}(u, x) \sim e^{-\frac{H}{T} x} \Psi\left(\frac{u+c T}{T^{H}}\right)
$$




\section{TECHNiCAL LEMMAS}

We first present a modification of Theorem 5.1 in [23], which is crucial for the proofs below. Then we present two lemmas related with the local behavior of the variance and correlation function of the investigated Gaussian process. Let $\left\{\xi_{u, k}(t), t \in E, k \in K_{u}\right\}$, with $K_{u}$ an index set and $E=[a, b]$ with $a \leq 0 \leq b$ be a family of centered continuous Gaussian random processes with variance function $\sigma_{\xi_{u, k}}^{2}$. We impose the following assumptions:

C0: Let $\left\{g_{k}(u), k \in K_{u}\right\}$ be a sequence of deterministic functions of $u$ satisfying

$$
\lim _{u \rightarrow \infty} \inf _{k \in K_{u}} g_{k}(u)=\infty
$$

C1: $\sigma_{\xi_{u, k}}(0)=1$ for all large $u$ and there exists a continuous function $h$ on $E$ such that

$$
\lim _{u \rightarrow \infty} \sup _{t \in E, k \in K_{u}}\left|g_{k}^{2}(u)\left(1-\sigma_{\xi_{u}, k}(t)\right)-h(t)\right|=0
$$

C2: There exists a centered Gaussian process with continuous trajectories and stationary increments $\zeta(t), t \in \mathbb{R}$, satisfying AI-AII, and

$$
\lim _{u \rightarrow \infty} \sup _{k \in K_{u}} \sup _{s \neq t, s, t \in E}\left|\frac{g_{k}^{2}(u)\left(1-\operatorname{Corr}\left(\xi_{u, k}(s), \xi_{u, k}(t)\right)\right)}{\frac{\sigma_{\zeta}^{2}(v(u) \Delta(u)|t-s|)}{\sigma_{\zeta}^{2}(\Delta(u))}}-1\right|=0,
$$

where $\Delta(u), v(u), u>0$ are positive functions such that

$$
\lim _{u \rightarrow \infty} \Delta(u)=\varphi \in[0, \infty], \quad \lim _{u \rightarrow \infty} v(u)=v \in[0, \infty) .
$$

Let, for given $x \geq 0$ and continuous function $h$

$$
\mathcal{B}_{\zeta_{\varphi}}^{h}(x, E)=\int_{\mathbb{R}} \mathbb{P}\left(\int_{E} \mathbb{I}_{0}\left(\sqrt{2} \zeta_{\varphi}(t)-\sigma_{\zeta_{\varphi}}^{2}(t)-h(t)+z\right) \mathrm{d} t>x\right) e^{-z} \mathrm{~d} z,
$$

with

$$
\zeta_{\varphi}(t)=\left\{\begin{array}{cc}
B_{2 \alpha_{0}}(v t) & \varphi=0 \\
\frac{\zeta(v \varphi t)}{\sigma_{\zeta}(\varphi)} & \varphi \in(0, \infty) . \\
B_{2 \alpha_{\infty}}(v t) & \varphi=\infty
\end{array}\right.
$$

In the special case $v=0, \zeta_{\varphi}(t) \equiv 0$. Note that by Borell-TIS Inequality [31, 32], we have

$$
\begin{aligned}
\mathcal{B}_{\zeta_{\varphi}}^{h}(x, E) & \leq \mathcal{B}_{\zeta_{\varphi}}^{h}(0, E) \\
& =\int_{\mathbb{R}} \mathbb{P}\left(\sup _{t \in E}\left(\sqrt{2} \zeta_{\varphi}(t)-\sigma_{\zeta_{\varphi}}^{2}(t)-h(t)\right)>z\right) e^{z} \mathrm{~d} z \\
& \leq e^{a}+\int_{a}^{\infty} e^{-\frac{(z-a)^{2}}{2 b}} e^{z} \mathrm{~d} z<\infty
\end{aligned}
$$

with

$$
a=\mathbb{E}\left(\sup _{t \in E}\left(\sqrt{2} \zeta_{\varphi}(t)-\sigma_{\zeta_{\varphi}}^{2}(t)-h(t)\right)\right)<\infty, \quad b=\sup _{t \in E} \operatorname{Var}\left(\sqrt{2} \zeta_{\varphi}(t)\right)<\infty .
$$

Lemma 4.1. Let $\left\{\xi_{u, k}(t), t \in E, k \in K_{u}\right\}$ with $E=[a, b]$ be a family of centered continuous Gaussian processes satisfying $\mathbf{C 1 - C 2}$. If $g_{k}(u), k \in K_{u}$ satisfies $\mathbf{C 0}$ and for any $x \geq 0$

$$
\mathbb{P}\left(\int_{E} \mathbb{I}_{0}\left(\xi_{u, k}(t)-g_{k}(u)\right) \mathrm{d} t>x\right)>0, \quad \forall k \in K_{u}
$$

then

$$
\lim _{u \rightarrow \infty} \sup _{k \in K_{u}}\left|\frac{\mathbb{P}\left(\int_{E} \mathbb{I}_{0}\left(\xi_{u, k}(t)-g_{k}(u)\right) \mathrm{d} t>x\right)}{\Psi\left(g_{k}(u)\right)}-\mathcal{B}_{\zeta_{\varphi}}^{h}(x, E)\right|=0
$$

holds for all $x \geq 0$. Additionally, $\mathcal{B}_{\zeta_{\varphi}}^{h}(x, E)$ is a continuous function over $[0$, mes $(E))$. 
Remark 4.2. i) In the special case $v=0$, for all $x \geq 0$, we have

$$
\lim _{u \rightarrow \infty} \sup _{k \in K_{u}}\left|\frac{\mathbb{P}\left(\int_{E} \mathbb{I}_{0}\left(\xi_{u, k}(t)-g_{k}(u)\right) \mathrm{d} t>x\right)}{\Psi\left(g_{k}(u)\right)}-\mathcal{B}_{0}^{h}(x, E)\right|=0
$$

ii) There exists a non-negative function $\rho$ which is a regularly varying function at 0 with index $2 \alpha_{0} \in(0,2]$ such that

$$
\lim _{u \rightarrow \infty} \sup _{k \in K_{u}} \sup _{s \neq t, s, t \in E}\left|\frac{g_{k}^{2}(u)\left(1-\operatorname{Corr}\left(\xi_{u, k}(s), \xi_{u, k}(t)\right)\right)}{\frac{\rho(v(u) \Delta(u)|t-s|)}{\rho(\Delta(u))}}-1\right|=0
$$

with $\Delta(u), v(u), u>0$ positive functions satisfying (32) for $\varphi=0$. If C2 is replaced by (35), then Lemma 4.1 still holds with $\zeta_{\varphi}(t)$ replaced by $B_{2 \alpha_{0}}(v t)$.

Proof of Lemma 4.1. In order to prove this lemma it suffices to check the conditions of Theorem 5.1 in [23]. That is we have to prove that (with $\left.\bar{\xi}_{u, k}(t)=\frac{\xi_{u, k}(t)}{\sigma_{\xi_{u, k}}(t)}\right)$

i) $\lim _{u \rightarrow \infty} \inf _{k \in K_{u}} g_{k}(u)=\infty$.

ii) $\sigma_{\xi_{u, k}}(0)=1$ for all large $u$ and any $k \in K_{u}$, and there exists some bounded continuous function $h$ on $E$ such that

$$
\lim _{u \rightarrow \infty} \sup _{t \in E, k \in K_{u}}\left|g_{k}^{2}(u)\left(1-\mathbb{E}\left\{\xi_{u, k}(t) \xi_{u, k}(0)\right\}\right)-\sigma_{\zeta_{\varphi}}^{2}(t)-h(t)\right|=0
$$

iii) For any $s, t \in E$,

$$
\lim _{u \rightarrow \infty} \sup _{k \in K_{u}}\left|g_{k}^{2}(u)\left(\operatorname{Var}\left(\bar{\xi}_{u, k}(t)-\bar{\xi}_{u, k}(s)\right)\right)-2 \operatorname{Var}\left(\zeta_{\varphi}(t)-\zeta_{\varphi}(s)\right)\right|=0 .
$$

iv) These exist positive constants $C, \nu, u_{0}$ such that

$$
\sup _{k \in K_{u}} g_{k}^{2}(u) \mathbb{E}\left\{\bar{\xi}_{u, k}(t)-\bar{\xi}_{u, k}(s)\right\}^{2} \leq C\|s-t\|^{\nu}
$$

holds for all $s, t \in E, u \geq u_{0}$.

It follows that i) holds straightforwardly from C0. Next we verify ii), iii) and iv).

We first prove iii). Uniform convergence theorem (see, e.g., [24]) gives that, for all $v \in[0, \infty)$ and $\varphi \in\{0, \infty\}$,

$$
\lim _{u \rightarrow \infty} \sup _{s, t \in E}\left|\frac{\sigma_{\zeta}^{2}(v(u) \Delta(u)|t-s|)}{\sigma_{\zeta}^{2}(\Delta(u))}-\operatorname{Var}\left(\zeta_{\varphi}(t)-\zeta_{\varphi}(s)\right)\right|=0 .
$$

For $v \in[0, \infty)$ and $\varphi \in(0, \infty)$, the above limit is still valid by the continuity of $\sigma_{\zeta_{\varphi}}^{2}$. Hence it follows from C2 that

$$
\lim _{u \rightarrow \infty} \sup _{k \in K_{u}} \sup _{s, t \in E}\left|g_{k}^{2}(u) \operatorname{Var}\left(\bar{\xi}_{u, k}(s)-\bar{\xi}_{u, k}(t)\right)-2 \operatorname{Var}\left(\zeta_{\varphi}(s)-\zeta_{\varphi}(t)\right)\right|=0
$$

This confirms that iii) is satisfied.

We next focus on iv). Let $f(t)=\frac{\sigma_{\zeta}^{2}(t)}{t^{\lambda}}$ with $0<\lambda<2 \min \left(\alpha_{0}, \alpha_{\infty}\right)$. Then $f$ is a regularly varying function at 0 and $\infty$ with index $2 \alpha_{0}-\lambda>0$ and $2 \alpha_{\infty}-\lambda>0$, respectively. By C2, we have that, for $u$ sufficiently large,

$$
\begin{aligned}
\sup _{k \in K_{u}} g_{k}^{2}(u) \mathbb{E}\left\{\left(\bar{\xi}_{u, k}(t)-\bar{\xi}_{u, k}(s)\right)^{2}\right\} & \leq 4 \frac{\sigma_{\zeta}^{2}(v(u) \Delta(u)|t-s|)}{\sigma_{\zeta}^{2}(\Delta(u))} \\
& \leq 4(v+1)^{\lambda} \frac{f(\Delta(u) v(u)|t-s|)}{f(\Delta(u))}|t-s|^{\lambda}, \quad s, t \in E .
\end{aligned}
$$

Uniform convergence theorem yields that for $0<\lambda<2 \min \left(\alpha_{0}, \alpha_{\infty}\right)$ and $\varphi \in\{0, \infty\}, v \in[0, \infty)$

$$
\lim _{u \rightarrow \infty} \sup _{s \neq t, s, t \in E}\left|\frac{f(\Delta(u) v(u)|t-s|)}{f(\Delta(u))}-(v|t-s|)^{\left(2 \alpha_{0}-\lambda\right) I_{\{\varphi=0\}}+\left(2 \alpha_{\infty}-\lambda\right) I_{\{\varphi=\infty\}}}\right|=0,
$$

which implies that for $u$ large enough

$$
\sup _{s \neq t, s, t \in E} \frac{f(\Delta(u) v(u)|t-s|)}{f(\Delta(u))}<\mathbb{Q} .
$$

with $\mathbb{Q}$ a positive constant. For $\varphi \in(0, \infty)$ and $v \in(0, \infty)$ the above inequality follows straightforwardly by noting the continuity of $f$. Hence for $u$ sufficiently large, for all $\varphi \in[0, \infty]$ and $v \in[0, \infty)$

$$
\sup _{k \in K_{u}} g_{k}^{2}(u) \mathbb{E}\left\{\left(\bar{\xi}_{u, k}(t)-\bar{\xi}_{u, k}(s)\right)^{2}\right\} \leq \mathbb{Q}|t-s|^{\lambda}, \quad s, t \in E
$$


with $0<\lambda<2 \min \left(\alpha_{0}, \alpha_{\infty}\right)$. This implies that iv) hold.

Finally, we prove ii). Notice that

$$
1-\mathbb{E}\left\{\xi_{u, k}(t) \xi_{u, k}(0)\right\}=\left(1-\sigma_{\xi_{u, k}}(t)\right)+\left(1-\operatorname{Corr}\left(\xi_{u, k}(t), \xi_{u, k}(0)\right)\right)-\left(1-\sigma_{\xi_{u, k}}(t)\right)\left(1-\operatorname{Corr}\left(\xi_{u, k}(t), \xi_{u, k}(0)\right)\right)
$$

In view of $(37)$,

$$
\lim _{u \rightarrow \infty} \sup _{t \in E, k \in K_{u}}\left|g_{k}^{2}(u)\left(1-\operatorname{Corr}\left(\xi_{u, k}(t), \xi_{u, k}(0)\right)\right)-\sigma_{\zeta_{\varphi}}^{2}(t)\right|=0
$$

which combined with $\mathbf{C 1}$ leads to

$$
\lim _{u \rightarrow \infty} \sup _{t \in E, k \in K_{u}}\left|g_{k}^{2}(u)\left(1-\operatorname{Corr}\left(\xi_{u, k}(t), \xi_{u, k}(0)\right)\right)\left(1-\sigma_{\xi_{u, k}}(t)\right)\right|=0 .
$$

Hence in view of $\mathbf{C 1}$ and the above limits,

$$
\lim _{u \rightarrow \infty} \sup _{t \in E, k \in K_{u}}\left|g_{k}^{2}(u)\left(1-\mathbb{E}\left\{\xi_{u, k}(t) \xi_{u, k}(0)\right\}\right)-\sigma_{\zeta_{\varphi}}^{2}(t)-h(t)\right|=0 .
$$

This confirms that ii) holds. Thus, applying Theorem 5.1 in [23], the claim is established for all the continuity points of $\mathcal{B}_{\zeta}^{h}(x, E)$.

Continuity of $\mathcal{B}_{\zeta}^{h}(x, E)$. Next we show that $\mathcal{B}_{\zeta}^{h}(x, E)$ is continuous over $[0$, mes $(E))$. Since $\mathcal{B}_{\zeta}^{h}(x, E)$ is right-continuous at 0 , then we are left with the continuity of $\mathcal{B}_{\zeta}^{h}(x, E)$ over $(0$, mes $(E))$. The claimed continuity at $x \in(0$, mes $(E))$ follows if

$$
\int_{\mathbb{R}} \mathbb{P}\left(\int_{E} \mathbb{I}\left(\sqrt{2} \zeta_{\varphi}(t)-\sigma_{\zeta_{\varphi}}^{2}(t)-h(t)+s>0\right) \mathrm{d} t=x\right) e^{-s} \mathrm{~d} s=0 .
$$

Assume that the probability space $\left(C(E), \mathcal{F}, \mathbb{P}^{*}\right)$ with $E=[a, b]$ is induced by the process $\left\{\sqrt{2} \zeta_{\varphi}(t)-\sigma_{\zeta_{\varphi}}^{2}(t)-h(t), t \in\right.$ $E\}$ with $C(E)$ denoting the collection of all continuous functions over $E$ and equipped with sup-normal, and $\mathcal{F}$ being the Borel $\sigma$-field on $C(E)$. Thus in order to complete the proof, it suffices to prove that for any $x \in(0, m e s(E))$

$$
\int_{\mathbb{R}} \mathbb{P}^{*}\left(\int_{E} \mathbb{I}(w(t)+s>0) \mathrm{d} t=x\right) e^{-s} \mathrm{~d} s=0
$$

where $w \in C(E)$. For any $x \in(0, \operatorname{mes}(E))$, let

$$
A_{s}=\left\{w \in C(E): \int_{E} \mathbb{I}(w(t)+s>0) \mathrm{d} t=x\right\}, \quad s \in \mathbb{R} .
$$

If $\int_{E} \mathbb{I}(w(t)+s>0) \mathrm{d} t=x$ with $x \in(0$, mes $(E))$, then $\inf _{t \in E} w(s) \leq-s<\sup _{t \in E} w(t)$. By the continuity of $w$, for $s<s^{\prime}$

This implies that

$$
\int_{E} \mathbb{I}\left(w(t)+s^{\prime}>0\right) \mathrm{d} t \in(x, \operatorname{mes}(E)]
$$

$$
A_{s} \cap A_{s^{\prime}}=\emptyset, \quad s \neq s^{\prime}, s, s^{\prime} \in \mathbb{R}
$$

Since $A_{s}, s \in \mathbb{R}$ are measurable sets and

$$
\sup _{\Lambda \subset \mathbb{R}, \# \Lambda<\infty} \sum_{s \in \Lambda} \mathbb{P}^{*}\left(A_{s}\right) \leq 1,
$$

where $\# \Lambda$ means the cardinality of set $\Lambda$, then

$$
\left\{s: s \in \mathbb{R} \quad \text { such that } \mathbb{P}^{*}\left(A_{s}\right)>0\right\}
$$

is a countable set, which indicates that

$$
\int_{\mathbb{R}} \mathbb{P}^{*}\left(A_{s}\right) e^{-s} \mathrm{~d} s=0 .
$$

Hence $\mathcal{B}_{\zeta_{\varphi}}^{h}(x, E)$ is continuous over $(0$, mes $(E))$. This completes the proof.

Let

$$
\sigma_{u}^{*}(t)=\operatorname{Var}^{1 / 2}\left(\frac{X(u t)}{u(1+c t)} M(u)\right), t \geq 0 \quad r_{u}(s, t)=\operatorname{Corr}\left(\frac{X(u s)}{u(1+c s)}, \frac{X(u t)}{u(1+c t)}\right), \quad s, t>0 .
$$

Assume that $\delta_{u}>0$ with $\lim _{u \rightarrow \infty} \delta_{u}=0$. The following lemma is due to [25]. 
Lemma 4.3. i) Suppose that $\mathbf{A I}$ is satisfied and let $t_{u}=\arg \inf _{t>0} \frac{u(1+c t)}{\sigma(u t)}$. If $u$ is large enough then $t_{u}$ is unique and $\lim _{u \rightarrow \infty} t_{u}=t^{*}=\frac{\alpha_{\infty}}{c\left(1-\alpha_{\infty}\right)}$. Moreover

$$
\lim _{u \rightarrow \infty} \sup _{t \in\left(t_{u}-\delta_{u}, t_{u}+\delta_{u}\right) \backslash\left\{t_{u}\right\}}\left|\frac{1-\sigma_{u}^{*}(t)}{\frac{B}{2 A}\left(t-t_{u}\right)^{2}}-1\right|=0,
$$

with $A, B$ defined in (24).

ii) If AI, AII hold, then

$$
\lim _{u \rightarrow \infty} \sup _{s \neq t, s, t \in\left(t_{u}-\delta_{u}, t_{u}+\delta_{u}\right)}\left|\frac{1-r_{u}(s, t)}{\frac{\sigma^{2}(u|s-t|)}{2 \sigma^{2}\left(u t^{*}\right)}}-1\right|=0 .
$$

Let next

$$
\widehat{\sigma}_{u}(t)=\operatorname{Var}^{1 / 2}\left(\frac{X(t)}{u+c t} m(u)\right), t \in[0, T], \quad \widehat{r}_{u}(s, t)=\operatorname{Corr}\left(\frac{X(s)}{u+c s}, \frac{X(t)}{u+c t}\right), s, t \in[0, T] .
$$

Lemma 4.4. i) If BI holds, then

$$
\lim _{u \rightarrow \infty} \sup _{t \in\left(T-\delta_{u}, T\right)}\left|\frac{1-\widehat{\sigma}_{u}(t)}{|T-t|}-\frac{\dot{\sigma}(T)}{\sigma(T)}\right|=0 .
$$

ii) If BII holds and $t=o(\sigma(t))$ as $t \rightarrow 0$, then

$$
\lim _{u \rightarrow \infty} \sup _{s \neq t, s, t \in\left(T-\delta_{u}, T\right)}\left|\frac{1-\widehat{r}_{u}(s, t)}{\frac{\sigma^{2}(|s-t|)}{2 \sigma^{2}(T)}-1}\right|=0 .
$$

\section{Proofs}

Hereafter, denote by $\mathbb{Q}, \mathbb{Q}_{i}, i=1,2,3, \ldots$ positive constants that may differ from line to line. The equivalence $f(u, S) \sim h(u)$ as $u \rightarrow \infty, S \rightarrow \infty$ means that $\lim _{S \rightarrow \infty} \lim _{u \rightarrow \infty} \frac{f(u, S)}{h(u)}=1$. Moreover, for any non-constant random variable $X$, denote by $\bar{X}:=\frac{X}{\sqrt{\operatorname{Var}(X)}}$.

Proof of Theorem 1.1 By the lack of upward jumps and using the strong Markov property, for any $x, u \geq 0$ we have

$$
\mathbb{P}\left(\int_{0}^{\infty} \mathbb{I}_{u}(X(t)-c t) \mathrm{d} t>x\right)=\mathbb{P}\left(\int_{0}^{\infty} \mathbb{I}_{0}(X(t)-c t) \mathrm{d} t>x\right) \mathbb{P}\left(\sup _{t \geq 0}(X(t)-c t) \geq u\right)
$$

and using e.g. [33][Theorem 3.3] we get $\mathbb{P}\left(\sup _{t \geq 0}(X(t)-c t) \geq u\right)=e^{-\alpha u}$, where $\alpha$ is the positive solution to the equation $\psi(\alpha)=c \alpha$ which by the strict convexity of $\psi$ and the assumption $\psi^{\prime}(0+)<c$ exists and is unique. This completes the proof.

Proof of Theorem 2.1 We consider each case i)-iii) separately. The following notation is valid for all three cases.

Put for $u>0, x \geq 0$ and $S>0$

$$
\begin{gathered}
\pi(u)=\mathbb{P}\left(\int_{E(u)} \mathbb{I}_{0}\left(Z_{u}(t)-n(u)\right) \mathrm{d} t>x\right), \\
\bar{Z}_{u, k}(t)=\bar{Z}_{u}(k S+t), \quad I_{k}=[k S,(k+1) S], \quad N_{1}(u)=\left[\frac{a_{1}(u)}{S}\right]-1, \quad N_{2}(u)=\left[\frac{a_{2}(u)}{S}\right]+1,
\end{gathered}
$$

and for some $\epsilon \in(0,1)$ set

Note that

$$
\begin{aligned}
& n_{u, k}^{-}=n(u)\left(1+(1-\epsilon) \inf _{t \in I_{k}} w(g(u)|t|)\right), \\
& n_{u, k}^{+}=n(u)\left(1+(1+\epsilon) \sup _{t \in I_{k}} w(g(u)|t|)\right) .
\end{aligned}
$$

$$
\begin{aligned}
\pi(u) & \leq \mathbb{P}\left(\sum_{k=N_{1}(u)-2}^{N_{2}(u)+2} \int_{I_{k}} \mathbb{I}_{0}\left(Z_{u}(t)-n(u)\right) \mathrm{d} t>x\right) \\
& \leq \mathbb{P}\left(\exists N_{1}(u)-2 \leq k \leq N_{2}(u)+2 \text { such that } \int_{I_{k}} \mathbb{I}_{0}\left(Z_{u}(t)-n(u)\right) \mathrm{d} t>x\right)
\end{aligned}
$$




$$
+\mathbb{P}\left(\exists N_{1}(u)-2 \leq k, l \leq N_{2}(u)+2, k \neq l \quad \text { such that } \int_{I_{k}} \mathbb{I}_{0}\left(Z_{u}(t)-n(u)\right) \mathrm{d} t>0, \int_{I_{l}} \mathbb{I}_{0}\left(Z_{u}(t)-n(u)\right) \mathrm{d} t>0\right)
$$

and

$$
\begin{aligned}
\pi(u) & \geq \mathbb{P}\left(\sum_{k=N_{1}(u)+2}^{N_{2}(u)-2} \int_{I_{k}} \mathbb{I}_{0}\left(Z_{u}(t)-n(u)\right) \mathrm{d} t>x\right) \\
& \geq \mathbb{P}\left(\exists N_{1}(u)+2 \leq k \leq N_{2}(u)-2 \text { such that } \int_{I_{k}} \mathbb{I}_{0}\left(Z_{u}(t)-n(u)\right) \mathrm{d} t>x\right) .
\end{aligned}
$$

Hence in view of (11) and using Bonferroni inequality, we have

$$
\pi^{+}(u, S)-\Sigma_{1}(u)-\Sigma_{2}(u) \leq \pi(u) \leq \pi^{-}(u, S)+\Sigma_{1}(u)+\Sigma_{2}(u)
$$

where

$$
\begin{aligned}
\pi^{ \pm}(u, S) & =\sum_{k=N_{1}(u) \pm 2}^{N_{2}(u) \mp 2} \mathbb{P}\left(\int_{I_{0}} \mathbb{I}_{0}\left(\bar{Z}_{u, k}(t)-n_{u, k}^{ \pm}\right) \mathrm{d} t>x\right), \\
\Sigma_{1}(u) & =\sum_{N_{1}(u)-2 \leq k \leq N_{2}(u)+1} \mathbb{P}\left(\sup _{t \in I_{k}} \bar{Z}_{u}(t)>n_{u, k}^{-}, \sup _{t \in I_{k+1}} \bar{Z}_{u}(t)>n_{u, k+1}^{-}\right), \\
\Sigma_{2}(u) & =\sum_{N_{1}(u)-2 \leq k<k+1<l \leq N_{2}(u)+1} \mathbb{P}\left(\sup _{t \in I_{k}} \bar{Z}_{u}(t)>n_{u, k}^{-}, \sup _{t \in I_{l}} \bar{Z}_{u}(t)>n_{u, l}^{-}\right) .
\end{aligned}
$$

$\Delta$ Case i) The idea of the proof is to divide $E(u)$ into a large number of tiny intervals for each of which we give a uniform exact asymptotics of sojourn times. For notational simplicity define

$$
\Theta(u):=\beta^{-1} \int_{y_{1}}^{y_{2}}|t|^{1 / \beta-1} e^{-|t|} \mathrm{d} t \frac{\overleftarrow{w}\left(n^{-2}(u)\right)}{g(u)} \Psi(n(u))
$$

Without loss of generality, we assume that $x_{1}>0, \lim _{u \rightarrow \infty} a_{1}(u)=\infty$ and $x_{2}>0, \lim _{u \rightarrow \infty} a_{2}(u)=-\infty$. Then by

$$
\left.y_{i}=x_{i} \mathbb{I}\left(x_{i}>0, \lim _{u \rightarrow \infty} a_{i}(u)=\infty\right\}\right)-x_{i} \mathbb{I}\left(x_{i}>0, \lim _{u \rightarrow \infty} a_{i}(u)=-\infty\right), i=1,2
$$

we have $y_{1}=x_{1}$ and $y_{2}=-x_{2}$. By (43), in order to complete the proof, it suffices to prove that, as $u \rightarrow \infty, S \rightarrow \infty$, $\pi^{-}(u, S) \sim \pi^{+}(u, S)$ and to show that $\Sigma_{i}(u)=o\left(\pi^{+}(u, S)\right), i=1,2$.

Analysis of $\pi^{ \pm}(u, S)$. We apply Lemma 4.1 to derive the uniform asymptotics for each term in the above sum. For this, we have to check conditions C0-C2. Following the notation in Lemma 4.1, let

$$
\xi_{u, k}(t)=\bar{Z}_{u, k}(t), \quad \sigma_{\xi_{u, k}}=1, \quad g_{k}(u)=n_{u, k}^{-}, \quad E=I_{0}, \quad K_{u}=\left\{k: N_{1}(u)-2 \leq k \leq N_{2}(u)+2\right\}
$$

Conditions C0-C1 hold straightforwardly with $h(t)=0$. By $(13)$, C2 holds for $\zeta=\eta, \nu(u)=1$ and $\Delta(u)$ given in (12). Hence we have that $\zeta_{\varphi}(t)=\eta_{\varphi}(t)$. Thus by Lemma 4.1 , we have that for $S>x$

$$
\lim _{u \rightarrow \infty} \sup _{k \in K_{u}}\left|\frac{\mathbb{P}\left(\int_{I_{0}} \mathbb{I}_{0}\left(\bar{Z}_{u, k}(t)-g_{k}(u)\right) \mathrm{d} t>x\right)}{\Psi\left(g_{k}(u)\right)}-\mathcal{B}_{\eta_{\varphi}}(x,[0, S])\right|=0
$$

Consequently,

$$
\begin{aligned}
\pi^{-}(u, S) & =\sum_{k=N_{1}(u)-2}^{N_{2}(u)+2} \mathbb{P}\left(\int_{I_{0}} \mathbb{I}_{0}\left(\bar{Z}_{u, k}(t)-n_{u, k}^{-}\right) \mathrm{d} t>x\right) \\
& \sim \sum_{k=N_{1}(u)-2}^{N_{2}(u)+2} \mathcal{B}_{\eta_{\varphi}}(x,[0, S]) \Psi\left(n_{u, k}^{-}\right) \\
& \sim \mathcal{B}_{\eta_{\varphi}}(x,[0, S]) \Psi(n(u)) \sum_{k=N_{1}(u)-2}^{N_{2}(u)+2} e^{-(1-\epsilon) n^{2}(u) \inf _{t \in I_{k}} w(g(u)|t|)}, \quad u \rightarrow \infty
\end{aligned}
$$


To get an upper bound for $\pi^{-}(u, S)$, it suffices to compute the sum above. Note that

$$
\lim _{u \rightarrow \infty} g(u)\left|a_{i}(u)\right|=0, \quad i=1,2
$$

Thus by Potter's theorem (see [24]) or Lemma 6.1 in [34], we have for any $0<\epsilon<\min (1, \beta)$ and all $u$ sufficiently large

$$
\frac{w(g(u)|s|)}{w(g(u)|t|)} \geq(1-\epsilon / 2) \min \left(\left|\frac{s}{t}\right|^{\beta-\epsilon},\left|\frac{s}{t}\right|^{\beta+\epsilon}\right), \quad s, t \in E(u), t \neq 0
$$

implying that for $u$ large enough

$$
\inf _{t \in I_{k}} w(g(u)|t|) \geq(1-\epsilon / 2)\left(\frac{|k|-1}{|k|}\right)^{\beta-\epsilon} \sup _{t \in I_{k}} w(g(u)|t|), \quad k \in K_{u}, k \neq 0 .
$$

Consequently, for any $0<\epsilon<1$, there exists $k_{\epsilon} \in \mathbb{N}$ such that for $|k| \geq k_{\epsilon}$ and $k \in K_{u}$ when $u$ is sufficiently large

$$
\inf _{t \in I_{k}} w(g(u)|t|) \geq(1-\epsilon) \sup _{t \in I_{k}} w(g(u)|t|)
$$

Hence, for $u$ large enough

$$
\begin{aligned}
\sum_{k=N_{1}(u)-2}^{N_{2}(u)+2} e^{-(1-\epsilon) n^{2}(u) \inf _{t \in I_{k}} w(g(u)|t|)} & \leq 2 k_{\epsilon}+\sum_{k \in K_{u},|k| \geq k_{\epsilon}} S^{-1} \int_{I_{k}} e^{-(1-\epsilon)^{2} n^{2}(u) w(g(u)|t|)} \mathrm{d} t \\
& \leq 2 k_{\epsilon}+S^{-1} \int_{a_{1}(u)}^{a_{2}(u)} e^{-(1-\epsilon)^{2} n^{2}(u) w(g(u)|t|)} \mathrm{d} t \\
& \leq 2 k_{\epsilon}+S^{-1}(g(u))^{-1} \int_{g(u) a_{1}(u)}^{g(u) a_{2}(u)} e^{-(1-\epsilon)^{2} n^{2}(u) w(|t|)} \mathrm{d} t
\end{aligned}
$$

We have that for $S>x$

$$
\int_{g(u) a_{1}(u)}^{g(u) a_{2}(u)} e^{-(1-\epsilon)^{2} n^{2}(u) w(|t|)} \mathrm{d} t \sim(1-\epsilon)^{-2 / \beta} \overleftarrow{w}\left(n^{-2}(u)\right) \beta^{-1} \int_{(1-\epsilon)^{2} y_{1}}^{(1-\epsilon)^{2} y_{2}}|t|^{1 / \beta-1} e^{-|t|} \mathrm{d} t .
$$

The proof of (48) is postponed to Appendix. Further by (45)-(48) we obtain for $S>x$

$$
\pi^{-}(u, S) \leq \frac{\mathcal{B}_{\eta_{\varphi}}(x,[0, S])}{S} \Theta(u)(1+o(1)), \quad u \rightarrow \infty
$$

and similarly,

$$
\pi^{+}(u, S) \geq \frac{\mathcal{B}_{\eta_{\varphi}}(x,[0, S])}{S} \Theta(u)(1+o(1)), \quad u \rightarrow \infty .
$$

$\underline{\text { Upper bound of } \Sigma_{i}(u), i=1,2}$. (44) with $x=0$ gives that

$$
\lim _{u \rightarrow \infty} \sup _{N_{1}(u)-2 \leq k \leq N_{2}(u)+2}\left|\frac{\mathbb{P}\left(\sup _{t \in I_{k}} \bar{Z}_{u}(t)>n_{u, k}^{-}\right)}{\Psi\left(n_{u, k}^{-}\right)}-\mathcal{B}_{\eta_{\varphi}}(0,[0, S])\right|=0 .
$$

Thus in light of (45)-(49) we have that

$$
\begin{aligned}
\Sigma_{1}(u) \leq & \sum_{N_{1}(u)-2 \leq k \leq N_{2}(u)+1}\left(\mathbb{P}\left(\sup _{t \in I_{k}} \bar{Z}_{u}(t)>n_{u, k}^{-}\right)+\mathbb{P}\left(\sup _{t \in I_{k+1}} \bar{Z}_{u}(t)>n_{u, k+1}^{-}\right)\right. \\
& \left.\quad-\mathbb{P}\left(\sup _{t \in I_{k} \cup I_{k+1}} \bar{Z}_{u}(t)>\widetilde{n}_{u, k}\right)\right) \\
\leq \quad & \sum_{\quad N_{1}(u)-2 \leq k \leq N_{2}(u)+1} \mathcal{B}_{\eta_{\varphi}}(0,[0, S]) \Psi\left(n_{u, k}^{-}\right)(1+o(1)) \\
& +\sum_{N_{1}(u)-2 \leq k \leq N_{2}(u)+1} \mathcal{B}_{\eta_{\varphi}}(0,[0, S]) \Psi\left(n_{u, k+1}^{-}\right)(1+o(1)) \\
& -\sum_{N_{1}(u)-2 \leq k \leq N_{2}(u)+1} \mathcal{B}_{\eta_{\varphi}}(0,[0,2 S]) \Psi\left(\widetilde{n}_{u, k}\right)(1+o(1)) \\
\leq \quad & S^{-1}\left(2 \mathcal{B}_{\eta_{\varphi}}(0,[0, S])-\mathcal{B}_{\eta_{\varphi}}(0,[0,2 S])\right) \Theta(u), \quad u \rightarrow \infty
\end{aligned}
$$


where $\widetilde{n}_{u, k}=\max \left(n_{u, k}^{-}, n_{u, k+1}^{-}\right)$. Moreover, by [21][Corollary 3.1] and (13) there exists $\mathcal{C}, \mathcal{C}_{1}>0$ such that for $N_{1}(u)-2 \leq k<k+1<l \leq N_{2}(u)+1$ and all $u$ large enough

$$
\mathbb{P}\left(\sup _{t \in I_{k}} \bar{Z}_{u}(t)>n_{u, k}^{-}, \sup _{t \in I_{l}} \bar{Z}_{u}(t)>n_{u, l}^{-}\right) \leq \mathcal{C} S^{2} e^{-\mathcal{C}_{1}|k-l|^{\gamma} S^{\gamma}} \Psi\left(\hat{n}_{u, k, l}\right),
$$

with $\hat{n}_{u, k, l}=\min \left(n_{u, k}^{-}, n_{u, l}^{-}\right)$and $\gamma=\min \left(\alpha_{0}, \alpha_{\infty}\right)$, which combined with (45)-(49) leads to

$$
\begin{aligned}
\Sigma_{2}(u) & \leq \sum_{N_{1}(u)-2 \leq k<k+1<l \leq N_{2}(u)+1} \mathcal{C} S^{2} e^{-\mathcal{C}_{1}|k-l|^{\gamma} S^{\gamma}} \Psi\left(\hat{n}_{u, k, l}\right) \\
& \leq \sum_{N_{1}(u)-2 \leq k \leq N_{2}(u)+1} \Psi\left(n_{u, k}^{-}\right) \sum_{l \geq 2} \mathcal{C} S^{2} e^{-\mathcal{C}_{1} l^{\gamma} S^{\gamma}} \\
& \leq \sum_{N_{1}(u)-2 \leq k \leq N_{2}(u)+1} \Psi\left(n_{u, k}^{-}\right) \mathbb{Q} S^{2} e^{-\mathbb{Q}_{1} S^{\gamma}} \\
& \leq \mathbb{Q} S^{2} e^{-\mathbb{Q}_{1} S^{\gamma}} \Theta(u), \quad u \rightarrow \infty .
\end{aligned}
$$

Consequently, by (52) and (54), for any $S>0$

$$
\Sigma_{1}(u)+\Sigma_{2}(u) \leq \mathbb{Q}\left(S^{-1}\left(2 \mathcal{B}_{\eta_{\varphi}}(0,[0, S])-\mathcal{B}_{\eta_{\varphi}}(0,[0,2 S])\right)+S^{2} e^{-\mathbb{Q}_{1} S^{\gamma}}\right) \Theta(u) .
$$

Exact asymptotics of $\pi(u)$. Inserting (49), (50) and (55) into (43) and dividing each term by $\Theta(u)$, we have that for $S>x$

$$
\begin{aligned}
\limsup _{u \rightarrow \infty} \frac{\pi(u)}{\Theta(u)} & \leq \frac{\mathcal{B}_{\eta_{\varphi}}(x,[0, S])}{S}+\mathbb{Q}\left(2 \frac{\mathcal{B}_{\eta_{\varphi}}(0,[0, S])}{S}-\frac{\mathcal{B}_{\eta_{\varphi}}(0,[0,2 S])}{S}+S^{2} e^{-\mathbb{Q}_{1} S^{\gamma}}\right) \\
\liminf _{u \rightarrow \infty} \frac{\pi(u)}{\Theta(u)} & \geq \frac{\mathcal{B}_{\eta_{\varphi}}(x,[0, S])}{S}-\mathbb{Q}\left(2 \frac{\mathcal{B}_{\eta_{\varphi}}(0,[0, S])}{S}-\frac{\mathcal{B}_{\eta_{\varphi}}(0,[0,2 S])}{S}+S^{2} e^{-\mathbb{Q}_{1} S^{\gamma}}\right) .
\end{aligned}
$$

Combination of (56)-(57) and the fact that (see [22] and [20])

$$
\lim _{S \rightarrow \infty} \frac{\mathcal{B}_{\eta_{\varphi}}(0,[0, S])}{S} \in(0, \infty)
$$

leads to

$$
\liminf _{S \rightarrow \infty} \frac{\mathcal{B}_{\eta_{\varphi}}(x,[0, S])}{S}=\limsup _{S \rightarrow \infty} \frac{\mathcal{B}_{\eta_{\varphi}}(x,[0, S])}{S}<\infty
$$

We next prove that

$$
\liminf _{S \rightarrow \infty} \frac{\mathcal{B}_{\eta_{\varphi}}(x,[0, S])}{S}>0
$$

Note that for $\mathbb{P}\left(\int_{E(u) \cap\left(\cup_{k \in \mathbb{Z}} I_{2 k}\right)} \mathbb{I}_{0}\left(Z_{u}(t)-n(u)\right) \mathrm{d} t>x\right)$, similarly as in (56)-(57) we have for $S>x$

$$
\liminf _{u \rightarrow \infty} \frac{\mathbb{P}\left(\int_{E(u) \cap\left(\cup_{k \in \mathbb{Z}} I_{2 k}\right)} \mathbb{I}_{0}\left(Z_{u}(t)-n(u)\right) \mathrm{d} t>x\right)}{\Theta(u)} \geq \frac{\mathcal{B}_{\eta_{\varphi}}(x,[0, S])}{2 S}-\mathbb{Q} S^{2} e^{-\mathbb{Q}_{1} S^{\gamma}},
$$

which together with (56) gives that that for any $S_{1}>x$

$$
\liminf _{S \rightarrow \infty} \frac{\mathcal{B}_{\eta_{\varphi}}(x,[0, S])}{S} \geq \frac{\mathcal{B}_{\eta_{\varphi}}\left(x,\left[0, S_{1}\right]\right)}{S_{1}}-\mathbb{Q} S_{1}^{2} e^{-\mathbb{Q}_{1} S_{1}^{\gamma}}
$$

Notice that for $S>x, \mathcal{B}_{\eta_{\varphi}}([0, S], x)$ is non-decreasing with respect to $S$ for $S>x$ and

$$
\mathcal{B}_{\eta_{\varphi}}([0, S], x) \geq \int_{\mathbb{R}} \mathbb{P}\left(\inf _{t \in[0, S]}\left(\sqrt{2} \eta_{\varphi}(t)-\operatorname{Var}\left(\eta_{\varphi}(t)\right)\right)>-z\right) e^{-z} \mathrm{~d} z>0 .
$$

Hence for some $S_{1}>x$,

$$
\liminf _{S \rightarrow \infty} \frac{\mathcal{B}_{\eta_{\varphi}}(x,[0, S])}{S} \geq \frac{\mathcal{B}_{\eta_{\varphi}}\left(x,\left[0, S_{1}\right]\right)}{S_{1}}-\mathbb{Q} S_{1}^{2} e^{-\mathbb{Q}_{1} S_{1}^{\gamma}}>0,
$$

which confirms that (58) holds. Hence we have

$$
\mathcal{B}_{\eta_{\varphi}}(x)=\lim _{S \rightarrow \infty} \frac{\mathcal{B}_{\eta_{\varphi}}(x,[0, S])}{S} \in(0, \infty) .
$$

Letting $S \rightarrow \infty$ in (56)-(57), we derive

$$
\pi(u) \sim \mathcal{B}_{\eta_{\varphi}}(x) \Theta(u), \quad u \rightarrow \infty
$$


This completes the proof of case i).

$\diamond$ Case ii) Let us first assume that

By (11) observe that for $u$ sufficiently large

$$
\lim _{u \rightarrow \infty} a_{1}(u)=-\infty, \quad \lim _{u \rightarrow \infty} a_{2}(u)=\infty .
$$

$$
p_{0}(u) \leq \pi(u) \leq p_{0}(u)+\pi_{1}(u)
$$

where

$$
p_{0}(u)=\mathbb{P}\left(\int_{[-S, S]} \mathbb{I}_{0}\left(Z_{u}(t)-n(u)\right) \mathrm{d} t>x\right), \quad \pi_{1}(u)=\sum_{k=N_{1}(u)-2, k \neq-1,0}^{N_{2}(u)+2} \mathbb{P}\left(\sup _{t \in I_{0}} \bar{Z}_{u, k}(t)>n_{u, k}^{-}\right) .
$$

In order to complete the proof, we shall derive the asymptotics of $p_{0}(u)$ and show further that

$$
\pi_{1}(u)=o\left(p_{0}(u)\right), \quad u \rightarrow \infty, S \rightarrow \infty .
$$

The analysis of $p_{0}(u)$. In order to apply Lemma 4.1, we need to check the validity of C0-C2. C0 holds straightforwardly. By (14) and uniform convergence theorem (e.g., in [24])

$$
\begin{aligned}
& \left.\lim _{u \rightarrow \infty} \sup _{t \in[-S, S]}\left|n^{2}(u) w(g(u)|t|)-\gamma\right| t\right|^{\beta} \mid \\
& \leq \lim _{u \rightarrow \infty} \sup _{t \in[-S, S]}\left|\left(n^{2}(u) w(g(u))-\gamma\right) \frac{w(g(u)|t|)}{w(g(u))}\right|+\left.\gamma \lim _{u \rightarrow \infty} \sup _{t \in[-S, S]}\left|\frac{w(g(u)|t|)}{w(g(u))}-\right| t\right|^{\beta} \mid=0 .
\end{aligned}
$$

By (11), (14) and uniform convergence theorem (e.g., in [24]), we have that for any $S>0$

$$
\begin{aligned}
\left.\lim _{u \rightarrow \infty} \sup _{t \in[-S, S]}\left|n^{2}(u)\left(1-\sigma_{u}(t)\right)-\gamma\right| t\right|^{\beta} \mid & \leq \lim _{u \rightarrow \infty} \sup _{t \in[-S, S]}\left|n^{2}(u) w(g(u)|t|)\right|\left|\frac{1-\sigma_{u}(t)}{w(g(u)|t|)}-1\right| \\
& \quad+\left.\lim _{u \rightarrow \infty} \sup _{t \in[-S, S]}\left|n^{2}(u) w(g(u)|t|)-\gamma\right| t\right|^{\beta} \mid \\
& =0,
\end{aligned}
$$

which confirms that $\mathbf{C 1}$ hold with $h(t)=\gamma|t|^{\beta}$. By $(13)$, C2 is satisfied with $\zeta_{\varphi}(t)=\eta_{\varphi}(t)$. Thus we have

$$
p_{0}(u) \sim \mathcal{B}_{\eta_{\varphi}}^{\gamma|t|^{\beta}}(x,[-S, S]) \Psi(n(u)) .
$$

Upper bound of $\pi_{1}(u)$. By (44)

$$
\begin{aligned}
\pi_{1}(u) & \sim \sum_{k=N_{1}(u)-2, k \neq-1,0}^{N_{2}(u)+2} \mathcal{B}_{\eta_{\varphi}}(0,[0, S]) \Psi\left(n_{u, k}^{-}\right) \\
& \leq \mathcal{B}_{\eta_{\varphi}}(0,[0, S]) \Psi(n(u)) \sum_{k=N_{1}(u)-2, k \neq-1,0}^{N_{2}(u)+2} e^{-(1-\epsilon) \inf _{t \in I_{k}} n^{2}(u) w(g(u)|t|)}, \quad u \rightarrow \infty .
\end{aligned}
$$

Further, using (46) for $u$ sufficiently large and $S>1$ we have

$$
\inf _{t \in I_{k}} n^{2}(u) w(g(u)|t|) \geq \frac{\gamma}{2} \inf _{t \in I_{k}} \frac{w(g(u)|t|)}{w(g(u))} \geq \mathbb{Q}(|k| S)^{\beta / 2}, \quad N_{1}(u)-2 \leq k \leq N_{2}(u)+2, k \neq-1,0 .
$$

Thus we have

$$
\begin{aligned}
\pi_{1}(u) & \leq \mathcal{B}_{\eta_{\varphi}}(0,[0, S]) \Psi(n(u)) \sum_{k=N_{1}(u)-2, k \neq-1,0}^{N_{2}(u)+2} e^{-\mathbb{Q}(|k| S)^{\beta / 2}} \\
& \leq \mathcal{B}_{\eta_{\varphi}}(0,[0, S]) \mathbb{Q}_{2} e^{-\mathbb{Q}_{1} S^{\beta / 2}} \Psi(n(u)), \quad u \rightarrow \infty
\end{aligned}
$$

Exact asymptotics of $\pi(u)$. Inserting (60) and (61) into (59) and dividing each terms by $\Psi(n(u))$, we have

$$
\begin{aligned}
\mathcal{B}_{\eta_{\varphi}}^{\gamma|t|^{\beta}}(x,[-S, S]) & \leq \liminf _{u \rightarrow \infty} \frac{\pi(u)}{\Psi(n(u))} \\
& \leq \limsup _{u \rightarrow \infty} \frac{\pi(u)}{\Psi(n(u))} \\
& \left.\leq \mathcal{B}_{\eta_{\varphi}|t|^{\beta}}^{\mid x}(x, S, S]\right)+\mathcal{B}_{\eta_{\varphi}}(0,[0, S]) \mathbb{Q}_{2} e^{-\mathbb{Q}_{1} S^{\beta / 2}} .
\end{aligned}
$$


This implies that for $S_{1}>x$

$$
0<\lim _{S \rightarrow \infty} \mathcal{B}_{\eta_{\varphi}}^{\gamma|t|^{\beta}}(x,[-S, S]) \leq \mathcal{B}_{\eta_{\varphi}|t|^{\beta}}^{\gamma}\left(x,\left[-S_{1}, S_{1}\right]\right)+\mathcal{B}_{\eta_{\varphi}}\left(0,\left[0, S_{1}\right]\right) \mathbb{Q}_{2} e^{-\mathbb{Q}_{1} S_{1}^{\beta / 2}}<\infty
$$

Moreover, by case i)

$$
\lim _{S \rightarrow \infty} \frac{\mathcal{B}_{\eta_{\varphi}}(0,[0, S])}{S} \in(0, \infty)
$$

Therefore, letting $S \rightarrow \infty$ in (62), we have

$$
\pi(u) \sim \mathcal{B}_{\eta_{\varphi}}^{\gamma|t|^{\beta}}(x,(-\infty, \infty)) \Psi(n(u)), \quad u \rightarrow \infty
$$

This establishes the claim for $a_{1}=-\infty, a_{2}=\infty$.

Next we focus on the case $a_{i} \in(-\infty, \infty), i=1,2$ with $a_{2}-a_{1}>x$. Note that for $\epsilon>0$ sufficiently small and $u$ sufficiently large

$$
\mathbb{P}\left(\int_{\left[a_{1}+\epsilon, a_{2}-\epsilon\right]} \mathbb{I}_{0}\left(Z_{u}(t)-n(u)\right) \mathrm{d} t>x\right) \leq \pi(u) \leq \mathbb{P}\left(\int_{\left[a_{1}-\epsilon, a_{2}+\epsilon\right]} \mathbb{I}_{0}\left(Z_{u}(t)-n(u)\right) \mathrm{d} t>x\right)
$$

Applying Lemma 4.1 it follows that

$$
\mathcal{B}_{\eta_{\varphi}}^{\gamma|t|^{\beta}}\left(x,\left[a_{1}+\epsilon, a_{2}-\epsilon\right]\right) \Psi(n(u)) \leq \pi(u) \leq \mathcal{B}_{\eta_{\varphi}}^{\gamma|t|^{\beta}}\left(x,\left[a_{1}-\epsilon, a_{2}+\epsilon\right]\right) \Psi(n(u)), \quad u \rightarrow \infty .
$$

Notice that

$$
\begin{aligned}
\mathcal{B}_{\eta_{\varphi}|t|^{\beta}}\left(x,\left[a_{1}-\epsilon, a_{2}+\epsilon\right]\right) & =\int_{\mathbb{R}} \mathbb{P}\left(\int_{\left[a_{1}-\epsilon, a_{2}+\epsilon\right]} \mathbb{I}_{0}(\widetilde{\eta}(t)+z) \mathrm{d} t>x\right) e^{-z} \mathrm{~d} z \\
& =\int_{\mathbb{R}} \mathbb{P}\left(\int_{\left[a_{1}, a_{2}\right]} \mathbb{I}_{0}(\widetilde{\eta}(t)+z) \mathrm{d} t+\int_{\left[a_{1}-\epsilon, a_{1}\right] \cup\left[a_{2}, a_{2}+\epsilon\right]} \mathbb{I}_{0}(\widetilde{\eta}(t)+z) \mathrm{d} t>x\right) e^{-z} \mathrm{~d} z
\end{aligned}
$$

where $\widetilde{\eta}(t)=\sqrt{2} \eta_{\varphi}(t)-\operatorname{Var}\left(\eta_{\varphi}(t)\right)-\gamma|t|^{\beta}$, and

$$
0 \leq \int_{\left[a_{1}-\epsilon, a_{1}\right] \cup\left[a_{2}, a_{2}+\epsilon\right]} \mathbb{I}_{0}(\widetilde{\eta}(t)+z) \mathrm{d} t \leq 2 \epsilon .
$$

Hence

$$
\mathcal{B}_{\eta_{\varphi}}^{\gamma|t|^{\beta}}\left(x,\left[a_{1}, a_{2}\right]\right) \leq \mathcal{B}_{\eta_{\varphi}}^{\gamma|t|^{\beta}}\left(x,\left[a_{1}-\epsilon, a_{2}+\epsilon\right]\right) \leq \mathcal{B}_{\eta_{\varphi}}^{\gamma|t|^{\beta}}\left(x-2 \epsilon,\left[a_{1}, a_{2}\right]\right) .
$$

The continuity of $\mathcal{B}_{\eta_{\varphi}}^{\gamma|t|^{\beta}}\left(x,\left[a_{1}, a_{2}\right]\right)$ ( see Lemma 4.1) leads to

$$
\lim _{\epsilon \rightarrow 0} \mathcal{B}_{\eta_{\varphi}|t|^{\beta}}\left(x-2 \epsilon,\left[a_{1}, a_{2}\right]\right)=\mathcal{B}_{\eta_{\varphi}}^{\gamma|t|^{\beta}}\left(x,\left[a_{1}, a_{2}\right]\right)
$$

implying that

$$
\lim _{\epsilon \rightarrow 0} \mathcal{B}_{\eta_{\varphi}}^{\gamma|t|^{\beta}}\left(x,\left[a_{1}-\epsilon, a_{2}+\epsilon\right]\right)=\mathcal{B}_{\eta_{\varphi}}^{\gamma|t|^{\beta}}\left(x,\left[a_{1}, a_{2}\right]\right)
$$

We can analogously show that

$$
\lim _{\epsilon \rightarrow 0} \mathcal{B}_{\eta_{\varphi}}^{\gamma|t|^{\beta}}\left(x,\left[a_{1}+\epsilon, a_{2}-\epsilon\right]\right)=\mathcal{B}_{\eta_{\varphi}}^{\gamma|t|^{\beta}}\left(x,\left[a_{1}, a_{2}\right]\right) .
$$

Letting $\epsilon \rightarrow 0$ in (63), we have that

$$
\pi(u) \sim \mathcal{B}_{\eta_{\varphi}}^{\gamma|t|^{\beta}}\left(x,\left[a_{1}, a_{2}\right]\right) \Psi(n(u)), \quad u \rightarrow \infty
$$

This establishes the claim for case $a_{i} \in(-\infty, \infty), i=1,2$ with $a_{2}-a_{1}>x$.

For the case that $a_{1}=-\infty, a_{2} \in(-\infty, \infty)$, and $a_{1} \in(-\infty, \infty), a_{2}=\infty$, we can establish the claim by using same approach.

$\diamond$ Case iii) Let us first consider the case that

$$
\lim _{u \rightarrow \infty} a_{1}(u) \frac{g(u)}{\overleftarrow{w}\left(n^{-2}(u)\right)}=-\infty, \quad \lim _{u \rightarrow \infty} a_{2}(u) \frac{g(u)}{\overleftarrow{w}\left(n^{-2}(u)\right)}=\infty
$$


By (11) we have

$$
p_{1}(u) \leq \pi_{2}(u) \leq \sum_{i=1}^{3} p_{i}(u)+\pi_{3}(u)
$$

where

$$
\begin{aligned}
& \pi_{2}(u)=\mathbb{P}\left(\int_{E(u)} \mathbb{I}_{0}\left(Z_{u}(t)-n(u)\right) \mathrm{d} t>\theta(u) x\right) \\
& p_{1}(u)=\mathbb{P}\left(\int_{[-\theta(u) S, \theta(u) S]} \mathbb{I}_{0}\left(Z_{u}(t)-n(u)\right) \mathrm{d} t>\theta(u) x\right), \\
& p_{i}(u)=\mathbb{P}\left(\sup _{t \in I_{i-3} \backslash[-\theta(u) S, \theta(u) S]} \bar{Z}_{u}(t)>n^{\prime}(u)\right), \quad i=2,3, \\
& \pi_{3}(u)=\sum_{k=N_{1}(u)-2, k \neq 0,-1}^{N_{2}(u)+2} \mathbb{P}\left(\sup _{t \in I_{k}} \bar{Z}_{u}(t)>n_{u, k}^{-}\right),
\end{aligned}
$$

with

$$
\theta(u)=\frac{\overleftarrow{w}\left(n^{-2}(u)\right)}{g(u)}, \quad n^{\prime}(u)=n(u)\left(1+(1-\epsilon) \inf _{t \in[-S, S] \backslash[-\theta(u) S, \theta(u) S]} w(g(u)|t|)\right), \quad 0<\epsilon<1
$$

We shall derive the exact asymptotics of $p_{1}(u)$ and then prove that $p_{2}(u), p_{3}(u)$ and $\pi_{3}(u)$ are all negligible compared with $p_{1}(u)$ as $u \rightarrow \infty$ and $S \rightarrow \infty$.

Analysis of $p_{1}(u)$. Substituting $t$ by $\theta(u) s$ we obtain

$$
p_{1}(u)=\mathbb{P}\left(\int_{[-S, S]} \mathbb{I}_{0}\left(Z_{u}(\theta(u) t)-n(u)\right) \mathrm{d} t>x\right) .
$$

Next we check C0-C2 in Lemma 4.1. C0 holds straightforwardly. Moreover, by (11) and uniform convergence theorem (e.g., in [24]), with noting that $n^{2}(u)=\left(w\left(\overleftarrow{w}\left(n^{-2}(u)\right)\right)\right)^{-1}$, we have that for any $S>0$

$$
\begin{aligned}
\left.\lim _{u \rightarrow \infty} \sup _{t \in[-S, S]}\left|n^{2}(u)\left(1-\sigma_{u}(\theta(u) t)\right)-\right| t\right|^{\beta} \mid \leq & \lim _{u \rightarrow \infty} \sup _{t \in[-S, S]}\left|\frac{w\left(\overleftarrow{w}\left(n^{-2}(u)\right)|t|\right)}{w\left(\overleftarrow{w}\left(n^{-2}(u)\right)\right)}\right|\left|\frac{1-\sigma_{u}(\theta(u) t)}{w\left(\overleftarrow{w}\left(n^{-2}(u)\right)|t|\right)}-1\right| \\
& \quad+\left.\lim _{u \rightarrow \infty} \sup _{t \in[-S, S]}\left|\frac{w\left(\overleftarrow{w}\left(n^{-2}(u)\right)|t|\right)}{w\left(\overleftarrow{w}\left(n^{-2}(u)\right)\right)}-\right| t\right|^{\beta} \mid \\
= & 0 .
\end{aligned}
$$

This confirms that $\mathbf{C} 1$ holds with $h(t)=|t|^{\beta}$. It follows from (13) that

$$
\lim _{u \rightarrow \infty} \sup _{s, t \in[-S, S], s \neq t}\left|\frac{n^{2}(u)\left(1-\operatorname{Corr}\left(\bar{Z}_{u}(\theta(u) t)-\bar{Z}_{u}(\theta(u) s)\right)\right)}{\frac{\sigma_{\eta}^{2}(\theta(u) \Delta(u)|t-s|)}{\sigma_{\eta}^{2}(\Delta(u))}}-1\right|=0
$$

with

$$
\lim _{u \rightarrow \infty} \theta(u)=\lim _{u \rightarrow \infty} \frac{\overleftarrow{w}\left(n^{-2}(u)\right)}{g(u)}=0
$$

This means that $\mathbf{C 2}$ is satisfied with $\zeta_{\varphi}=0$. Therefore we have that

$$
p_{1}(u) \sim \mathcal{B}_{0}^{|t|^{\beta}}(x,[-S, S]) \Psi(n(u)) .
$$

Upper bound for $\pi_{3}(u), p_{i}(u), i=2,3$. Next we find an upper bound of $\pi_{3}(u)$. Similarly as (61), by (44), we have

$$
\pi_{3}(u) \leq \mathcal{B}_{\eta_{\varphi}}(0,[0, S]) \mathbb{Q}_{2} e^{-\mathbb{Q}_{1} S^{\beta / 2}} \Psi(n(u)), \quad u \rightarrow \infty .
$$

Finally, we focus on deriving an upper bound of $p_{i}(u), i=2,3$. In light of (44), we have that

$$
p_{i}(u) \leq \mathbb{P}\left(\sup _{t \in I_{i-3}} \bar{Z}_{u}(t)>n^{\prime}(u)\right) \sim \mathcal{B}_{\eta_{\varphi}}(0,[0, S]) \Psi\left(n^{\prime}(u)\right), \quad i=2,3 .
$$

By (46), we have that

$n^{2}(u) \inf _{t \in[-S, S] \backslash[-\theta(u) S, \theta(u)) S]} w(g(u)|t|) \geq \frac{1}{2} \inf _{t \in[0, S / \theta(u)] \backslash[-S, S]} \frac{w\left(\overleftarrow{w}\left(n^{-2}(u)\right)|t|\right)}{w\left(\overleftarrow{w}\left(n^{-2}(u)\right)\right)} \geq \frac{1}{4} \inf _{t \in[0, S / \theta(u)] \backslash[-S, S]}|t|^{\beta / 2} \geq \frac{1}{4}|S|^{\beta / 2}$ 
which together with (69) leads to

$$
p_{i}(u) \leq \mathcal{B}_{\eta_{\varphi}}(0,[0, S]) e^{-\mathbb{Q}|S|^{\beta / 2} / 8} \Psi(n(u)), \quad u \rightarrow \infty, i=2,3
$$

Exact asymptotics of $\pi_{2}(u)$. Inserting (67), (68) and (70) into (66), we have that

$$
\begin{aligned}
\liminf _{u \rightarrow \infty} \frac{\pi_{2}(u)}{\Psi(n(u))} \geq \mathcal{B}_{0}^{|t|^{\beta}}(x,[-S, S]), \\
\limsup _{u \rightarrow \infty} \frac{\pi_{2}(u)}{\Psi(n(u))} \leq \mathcal{B}_{0}^{|t|^{\beta}}(x,[-S, S])+\mathcal{B}_{\eta_{\varphi}}(0,[0, S]) e^{-\mathbb{Q} S^{\beta / 2}},
\end{aligned}
$$

which implies that

$$
0<\lim _{S \rightarrow \infty} \mathcal{B}_{0}^{|t|^{\beta}}(x,[-S, S]) \leq \mathcal{B}_{0}^{|t|^{\beta}}\left(x,\left[-S_{1}, S_{1}\right]\right)+\mathcal{B}_{\eta_{\varphi}}\left(0,\left[0, S_{1}\right]\right) e^{-\mathbb{Q} S_{1}^{\beta / 2}}<\infty, \quad S_{1}>x .
$$

Letting $S \rightarrow \infty$ in (71) yields that

$$
\pi_{2}(u) \sim \mathcal{B}_{0}^{|t|^{\beta}}(x,(-\infty, \infty)) \Psi(n(u))
$$

This establishes the claim for $b_{1}=-\infty, b_{2}=\infty$.

Next we focus on the case $b_{1}, b_{2} \in(-\infty, \infty)$ with $b_{2}-b_{1}>x$. For $\epsilon>0$ sufficiently small and $u$ sufficiently large, we have

$\mathbb{P}\left(\int_{\left[\theta(u)\left(b_{1}+\epsilon\right), \theta(u)\left(b_{2}-\epsilon\right)\right]} \mathbb{I}_{0}\left(Z_{u}(t)-n(u)\right) \mathrm{d} t>\theta(u) x\right) \leq \pi_{2}(u) \leq \mathbb{P}\left(\int_{\left[\theta(u)\left(b_{1}-\epsilon\right), \theta(u)\left(b_{2}+\epsilon\right)\right]} \mathbb{I}_{0}\left(Z_{u}(t)-n(u)\right) \mathrm{d} t>\theta(u) x\right)$.

Applying Lemma 4.1, similarly as in (67), we derive that

$$
\mathcal{B}_{0}^{|t|^{\beta}}\left(x,\left[b_{1}+\epsilon, b_{2}-\epsilon\right]\right) \Psi(n(u)) \leq \pi_{2}(u) \leq \mathcal{B}_{0}^{|t|^{\beta}}\left(x,\left[b_{1}-\epsilon, b_{2}+\epsilon\right]\right) \Psi(n(u)), \quad u \rightarrow \infty .
$$

In light of (64) and (65), letting $\epsilon \rightarrow 0$ in above inequality, we get

$$
\pi_{2}(u) \sim \mathcal{B}_{0}^{|t|^{\beta}}\left(x,\left[b_{1}, b_{2}\right]\right) \Psi(n(u)), \quad u \rightarrow \infty .
$$

Hence the claim for case $b_{1}, b_{2} \in(-\infty, \infty)$ is established.

The proofs of claims for other cases of $b_{1}$ and $b_{2}$ can be done by the same line of reasoning.

This completes the proof.

Proof of Theorem 3.1 We set $\Delta(u)=\overleftarrow{\sigma}\left(\frac{\sqrt{2} \sigma^{2}\left(u t^{*}\right)}{u\left(1+c t^{*}\right)}\right)$. By $(23)$ observe that for any $u>0$ (recall the definition of $\overleftarrow{\sigma}$ in $(22))$

$$
\pi_{4}(u) \leq \mathbb{P}\left(\frac{1}{\Delta(u)} \int_{0}^{\infty} \mathbb{I}_{u}(X(t)-c t) \mathrm{d} t>x\right) \leq \pi_{4}(u)+\pi_{5}(u)
$$

where

$$
\begin{aligned}
& \pi_{4}(u)=\mathbb{P}\left(\frac{u}{\Delta(u)} \int_{E_{1}(u)} \mathbb{I}_{0}\left(\frac{X(u t)}{u(1+c t)} M(u)-M(u)\right) \mathrm{d} t>x\right), \\
& \pi_{5}(u)=\mathbb{P}\left(\sup _{t \in[0, \infty] \backslash E_{1}(u)} \frac{X(u t)}{u(1+c t)} M(u)>M(u)\right),
\end{aligned}
$$

with

$$
E_{1}(u)=\left[t_{u}-\frac{\ln M(u)}{M(u)}, t_{u}+\frac{\ln M(u)}{M(u)}\right] .
$$

The idea of the proof is to derive the exact asymptotics of $\pi_{4}(u)$ using Theorem 2.1 and to show that $\pi_{5}(u)=o\left(\pi_{4}(u)\right)$ as $u \rightarrow \infty$.

The analysis of $\pi_{4}(u)$. Scaling time with $\frac{u}{\Delta(u)}$, we have

$$
\pi_{4}(u)=\mathbb{P}\left(\int_{E_{2}(u)} \mathbb{I}_{0}\left(Z_{u}(t)-M(u)\right) \mathrm{d} t>x\right)
$$

where

$$
Z_{u}(t)=\frac{X\left(u t_{u}+\Delta(u) t\right)}{u\left(1+c t_{u}\right)+c \Delta(u) t}, \quad E_{2}(u)=\left[-\frac{u \ln M(u)}{\Delta(u) M(u)}, \frac{u \ln M(u)}{\Delta(u) M(u)}\right]
$$


It follows from (39) and (40) that

$$
\begin{aligned}
& 1-\sqrt{\operatorname{Var}\left(Z_{u}(t)\right)} \sim\left(\sqrt{\left.\frac{B}{2 A} \frac{\Delta(u)}{u}|t|\right)^{2}, \quad t \in E_{2}(u),}\right. \\
& \lim _{u \rightarrow \infty} \sup _{s \neq t, s, t \in E_{2}(u)}\left|M^{2}(u) \frac{1-\operatorname{Corr}\left(Z_{u}(s), Z_{u}(t)\right)}{\frac{\sigma^{2}(\Delta(u)|s-t|)}{\sigma^{2}(\Delta(u))}}-1\right|=0,
\end{aligned}
$$

which imply that (11) and (13) hold with

$$
\begin{aligned}
w(t) & =t^{2}, \quad g(u)=\sqrt{\frac{B}{2 A} \frac{\Delta(u)}{u}}, \quad n(u)=M(u), \quad \eta=X, \quad \Delta(u)=\overleftarrow{\sigma}\left(\frac{\sqrt{2} \sigma^{2}\left(u t^{*}\right)}{u\left(1+c t^{*}\right)}\right) \\
a_{1}(u) & =-\frac{u \ln M(u)}{\Delta(u) M(u)}, \quad a_{2}(u)=\frac{u \ln M(u)}{\Delta(u) M(u)}
\end{aligned}
$$

Next we check the assumptions of i) in Theorem 2.1. Following the notation in Theorem 2.1, we have

$$
\lim _{u \rightarrow \infty} g(u)=\lim _{u \rightarrow \infty} \sqrt{\frac{B}{2 A}} \frac{\Delta(u)}{u}=0, \quad \lim _{u \rightarrow \infty} g(u)\left|a_{i}(u)\right|=\lim _{u \rightarrow \infty} \sqrt{\frac{B}{2 A}} \frac{\ln M(u)}{M(u)}=0, i=1,2 .
$$

Note that

$$
n^{2}(u) w(g(u))=\frac{B}{2 A}\left(\frac{M(u) \Delta(u)}{u}\right)^{2} \sim \mathbb{Q}\left(\frac{\overleftarrow{\sigma}\left(u^{-1} \sigma^{2}(u)\right)}{\sigma(u)}\right)^{2}
$$

is a regularly varying function at $\infty$ with index $2 \tau$ with

$$
\tau=\left\{\begin{array}{cc}
\frac{2 \alpha_{\infty}-1}{\alpha_{0}}-\alpha_{\infty}, & \varphi=0 \\
-\alpha_{\infty}, & \varphi \in(0, \infty) \\
\frac{2 \alpha_{\infty}-1}{\alpha_{\infty}}-\alpha_{\infty}, & \varphi=\infty
\end{array}\right.
$$

Since $\tau<0$ for all $\varphi$, then for all $\varphi \in[0, \infty]$

$$
\gamma=\lim _{u \rightarrow \infty} n^{2}(u) w(g(u))=0
$$

Moreover,

$$
\lim _{u \rightarrow \infty} n^{2}(u) w\left(g(u)\left|a_{i}(u)\right|\right)=\lim _{u \rightarrow \infty} \frac{B}{2 A}(\ln M(u))^{2}=\infty, \quad i=1,2
$$

and by (80)

$$
\lim _{u \rightarrow \infty} a_{1}(u)=-\lim _{u \rightarrow \infty} \frac{u \ln M(u)}{\Delta(u) M(u)}=-\mathbb{Q} \lim _{u \rightarrow \infty} \frac{\sigma(u) \ln M(u)}{\overleftarrow{\sigma}\left(u^{-1} \sigma^{2}(u)\right)}=-\infty,
$$

which imply that $y_{1}=-\infty$. Similarly we can check that $y_{2}=\infty$. Additionally,

$$
\lim _{u \rightarrow \infty} n(u) w\left(g(u)\left|a_{i}(u)\right|\right)=\frac{B}{A} \lim _{u \rightarrow \infty} \frac{(\ln M(u))^{2}}{M(u)}=0, \quad i=1,2 .
$$

Hence all the assumptions in i) of Theorem 2.1 are satisfied, leading to

$$
\begin{aligned}
\pi_{4}(u) & \sim \mathcal{B}_{X_{\varphi}}(x) \frac{1}{2} \int_{-\infty}^{\infty}|t|^{1 / 2-1} e^{-|t|} \mathrm{d} t \sqrt{\frac{2 A}{B}} \frac{u}{M(u) \Delta(u)} \Psi(M(u)) \\
& \sim \mathcal{B}_{X_{\varphi}}(x) \sqrt{\frac{2 A \pi}{B}} \frac{u}{M(u) \Delta(u)} \Psi(M(u)), \quad u \rightarrow \infty
\end{aligned}
$$

where $X_{\varphi}$ is given in (25).

Upper bound of $\pi_{5}(u)$. By [20][Lemma 7] or [25][Lemma 5.6], we have

$$
\pi_{5}(u)=o\left(\frac{u}{M(u) \Delta(u)} \Psi(M(u))\right), \quad u \rightarrow \infty,
$$

which combined with (72) and (85) leads to

$$
\mathbb{P}\left(\frac{1}{\Delta(u)} \int_{0}^{\infty} \mathbb{I}_{u}(X(t)-c t) \mathrm{d} t>x\right) \sim \mathcal{B}_{X_{\varphi}}(x) \sqrt{\frac{2 A \pi}{B}} \frac{u}{M(u) \Delta(u)} \Psi(M(u)), \quad u \rightarrow \infty
$$


This completes the proof.

Proof of Corollary 3.3 We also set $\Delta(u)=\overleftarrow{\sigma}\left(\frac{\sqrt{2} \sigma^{2}\left(u t^{*}\right)}{u\left(1+c t^{*}\right)}\right)$. Observe that for $0 \leq x_{1} \leq x_{2}<\infty$ and $u>0$ the conditional distribution can be rewritten as the ratio of two sojourn probabilities over different intervals, i.e.,

$$
\mathbb{P}\left(\frac{\tau_{u}^{*}\left(x_{1}, x_{2}\right)-u t_{u}}{A(u)}<y\right)=\frac{q(u)}{\mathbb{P}\left(\frac{1}{\Delta(u)} \int_{0}^{\infty} \mathbb{I}_{u}(X(t)-c t) \mathrm{d} t>x_{1}\right)},
$$

where $y \in \mathbb{R}$ and

$$
q(u)=\mathbb{P}\left(\frac{1}{\Delta(u)} \int_{\left[0, u t_{u}+A(u) y\right]} \mathbb{I}_{u}(X(t)-c t) \mathrm{d} t>x_{2}\right) .
$$

Hence in order to complete the proof it suffices to derive the asymptotics of $q(u)$. Using notation for $E_{1}(u)$ and $\pi_{5}(u)$ introduced in (73) and (74), we have that

$$
q_{1}(u) \leq q(u) \leq q_{1}(u)+\pi_{4}(u)
$$

with

$$
q_{1}(u)=\mathbb{P}\left(\frac{u}{\Delta(u)} \int_{\left[t_{u}-\frac{\ln M(u)}{M(u)}, t_{u}+\frac{A(u)}{u} y\right]} \mathbb{I}_{0}\left(\frac{X(u t)}{u(1+c t)} M(u)-M(u)\right) \mathrm{d} t>x_{2}\right) .
$$

Scaling time by $\frac{u}{\Delta(u)}$, we rewrite

$$
q_{1}(u)=\mathbb{P}\left(\int_{E_{3}(u)} \mathbb{I}_{0}\left(Z_{u}(t)-M(u)\right) \mathrm{d} t>x_{2}\right),
$$

where

$$
Z_{u}(t)=\frac{X\left(u t_{u}+\Delta(u) t\right)}{u\left(1+c t_{u}\right)+c \Delta(u) t}, \quad E_{3}(u)=\left[-\frac{u \ln M(u)}{\Delta(u) M(u)}, \frac{A(u)}{\Delta(u)} y\right] .
$$

Using the same notation as introduced in (78) with the exception that $a_{2}(u)=\frac{A(u)}{\Delta(u)} y$, we have that (77), (79), (81) and (84) also hold. We next get the value of $y_{i}, i=1,2$. For this, note that

$$
\lim _{u \rightarrow \infty} n^{2}(u) w\left(g(u)\left|a_{2}(u)\right|\right)=\lim _{u \rightarrow \infty} \frac{B}{2 A}\left(\frac{M(u) A(u)}{u} y\right)^{2}=\frac{\alpha_{\infty}}{c^{2}\left(1-\alpha_{\infty}\right)^{3}} \frac{B}{2 A} y^{2}=\frac{y^{2}}{2},
$$

where $A$ and $B$ are given in (24). Moreover, by (80)

$$
\lim _{u \rightarrow \infty} \frac{A(u)}{\Delta(u)}=\mathbb{Q} \lim _{u \rightarrow \infty} \frac{\sigma(u)}{\bar{\sigma}\left(u^{-1} \sigma^{2}(u)\right)}=\infty
$$

Hence $y_{2}=\frac{y^{2}}{2} I_{\{y>0\}}-\frac{y^{2}}{2} I_{\{y<0\}}$. Additionally, it follows from (82)-(83) that $y_{1}=-\infty$. Thus applying i) in Theorem 2.1 we have

$$
\begin{aligned}
q_{1}(u) & \sim \mathcal{B}_{X_{\varphi}}\left(x_{2}\right) \sqrt{\frac{2 A \pi}{B}} \frac{1}{2 \sqrt{\pi}} \int_{-\infty}^{y_{2}}|t|^{-1 / 2} e^{-|t|} d t \frac{u}{M(u) \Delta(u)} \Psi(M(u)) \\
& \sim \mathcal{B}_{X_{\varphi}}\left(x_{2}\right) \sqrt{\frac{2 A \pi}{B}} \Phi(y) \frac{u}{M(u) \Delta(u)} \Psi(M(u)), \quad u \rightarrow \infty .
\end{aligned}
$$

Combination of (86), (88) and (89) leads to

$$
q(u) \sim \mathcal{B}_{X_{\varphi}}\left(x_{2}\right) \sqrt{\frac{2 A \pi}{B}} \Phi(y) \frac{u}{M(u) \Delta(u)} \Psi(M(u)), \quad u \rightarrow \infty .
$$

Consequently, by Theorem 3.1 and (87)

$$
\mathbb{P}\left(\frac{\tau_{u}^{*}\left(x_{1}, x_{2}\right)-u t_{u}}{A(u)}<y\right)=\frac{\mathcal{B}_{X_{\varphi}}\left(x_{2}\right)}{\mathcal{B}_{X_{\varphi}}\left(x_{1}\right)} \Phi(y), \quad u \rightarrow \infty
$$

establishing the proof.

Proof of Theorem 3.4 We set $\Delta(u)=\overleftarrow{\sigma}\left(\frac{\sqrt{2} \sigma^{2}(T)}{u+c T}\right)$. Following (26), we have

$$
\lambda(u) \leq \mathbb{P}\left(v(u) \int_{0}^{T} \mathbb{I}_{u}(X(t)-c t) \mathrm{d} t>x\right) \leq \lambda(u)+\lambda_{1}(u),
$$


where

$$
\begin{aligned}
\lambda(u) & =\mathbb{P}\left(v(u) \int_{E_{4}(u)} \mathbb{I}_{0}\left(\frac{X(t)}{u+c t} m(u)-m(u)\right) \mathrm{d} t>x\right), \\
\lambda_{1}(u) & =\mathbb{P}\left(\sup _{t \in[0, T] \backslash E_{4}(u)} \frac{X(t)}{u+c t} m(u)>m(u)\right),
\end{aligned}
$$

with

$$
E_{4}(u)=\left[T-\left(\frac{\ln m(u)}{m(u)}\right)^{2}, T\right], \quad v(u)=1 / \Delta(u)
$$

if $\lim _{t \rightarrow 0} \frac{|t|}{\sigma^{2}(|t|)} \in[0, \infty)$ and

$$
v(u)=(m(u))^{2}
$$

if $\lim _{t \rightarrow 0} \frac{|t|}{\sigma^{2}(|t|)}=\infty$. We shall derive the exact asymptotics of $\lambda(u)$ by applying Theorem 2.1, and show that

$$
\lambda_{1}(u)=o(\lambda(u)), \quad u \rightarrow \infty .
$$

We distinguish three cases: $\lim _{t \rightarrow 0} \frac{|t|}{\sigma^{2}(|t|)}=0,(0, \infty)$ and $\infty$, respectively.

Case $\lim _{t \rightarrow 0} \frac{|t|}{\sigma^{2}(|t|)}=0$.

Asymptotics of $\lambda(u)$. Noting that $v(u)=1 / \Delta(u)$ and scaling time by $\Delta(u)$ we get

$$
\lambda(u)=\mathbb{P}\left(\int_{E_{5}(u)} \mathbb{I}_{0}\left(Z_{u}(t)-m(u)\right) \mathrm{d} t>x\right),
$$

with

$$
Z_{u}(t)=\frac{X(T-\Delta(u) t)}{u+c T-c \Delta(u) t} m(u), \quad E_{5}(u)=\left[0,(\Delta(u))^{-1}\left(\frac{\ln m(u)}{m(u)}\right)^{2}\right] .
$$

In light of Lemma 4.4, we have that

$$
\begin{aligned}
& 1-\sqrt{\operatorname{Var}\left(Z_{u}(t)\right)} \sim \frac{\dot{\sigma}(T)}{\sigma(T)} \Delta(u)|t|, t \in E_{5}(u), \\
& \lim _{u \rightarrow \infty} \sup _{s \neq t, s, t \in E_{5}(u)}\left|\frac{m^{2}(u)\left(1-\operatorname{Corr}\left(Z_{u}(t), Z_{u}(s)\right)\right)}{\frac{\sigma^{2}(\Delta(u)|t-s|)}{\sigma^{2}(\Delta(u))}}-1\right|=0 .
\end{aligned}
$$

With the notation introduced in Theorem 2.1, (93) implies that

$$
\begin{aligned}
& n(u)=m(u), \quad w=t, \quad g(u)=\frac{\dot{\sigma}(T)}{\sigma(T)} \Delta(u), \quad \Delta(u)=\overleftarrow{\sigma}\left(\frac{\sqrt{2} \sigma^{2}(T)}{u+c T}\right), \quad \eta=X, \\
& E(u)=\left[a_{1}(u), a_{2}(u)\right], \quad a_{1}(u)=0, \quad a_{2}(u)=(\Delta(u))^{-1}\left(\frac{\ln m(u)}{m(u)}\right)^{2} .
\end{aligned}
$$

Next we check the conditions in i) of Theorem 2.1. It follows that

$$
\lim _{u \rightarrow \infty} g(u)=\lim _{u \rightarrow \infty} \frac{\dot{\sigma}(T)}{\sigma(T)} \Delta(u)=0, \quad g(u) a_{1}(u)=0, \quad \lim _{u \rightarrow \infty} g(u) a_{2}(u)=\lim _{u \rightarrow \infty}\left(\frac{\ln m(u)}{m(u)}\right)^{2}=0,
$$

and

Moreover,

$$
\gamma=\lim _{u \rightarrow \infty} n^{2}(u) w(g(u))=\lim _{u \rightarrow \infty} \frac{\dot{\sigma}(T)}{\sigma(T)} \Delta(u)(m(u))^{2}=\mathbb{Q} \lim _{u \rightarrow \infty} \frac{\overleftarrow{\sigma}\left(u^{-1}\right)}{\sigma^{2}\left(\overleftarrow{\sigma}\left(u^{-1}\right)\right)}=0
$$

$$
\begin{gathered}
x_{1}=\lim _{u \rightarrow \infty} n^{2}(u) w\left(g(u)\left|a_{1}(u)\right|\right)=0, \quad x_{2}=\lim _{u \rightarrow \infty} n^{2}(u) w\left(g(u)\left|a_{2}(u)\right|\right)=\mathbb{Q} \lim _{u \rightarrow \infty} \frac{\dot{\sigma}(T)}{\sigma(T)}(\ln m(u))^{2}=\infty, \\
\lim _{u \rightarrow \infty} a_{2}(u)=\mathbb{Q} \lim _{u \rightarrow \infty} \frac{\sigma^{2}\left(\overleftarrow{\sigma}\left(u^{-1}\right)\right)}{\overleftarrow{\sigma}\left(u^{-1}\right)} \ln m(u)=\infty,
\end{gathered}
$$

imply that $y_{1}=0$ and $y_{2}=\infty$. Additionally,

$$
\lim _{u \rightarrow \infty} n(u)\left(w\left(g(u)\left|a_{1}(u)\right|\right)+w\left(g(u)\left|a_{2}(u)\right|\right)\right)=\lim _{u \rightarrow \infty} \frac{\dot{\sigma}(T)}{\sigma(T)} \frac{(\ln m(u))^{2}}{m(u)}=0, \quad \lim _{u \rightarrow \infty} \Delta(u)=0 .
$$


Consequently, by i) of Theorem 2.1 and Remark 2.2 we have

$$
\lambda(u) \sim \mathcal{B}_{B_{2 \alpha_{0}}}(x) \frac{\sigma(T)}{\dot{\sigma}(T)} \frac{1}{(m(u))^{2} \Delta(u)} \Psi(m(u)) .
$$

Upper bound of $\lambda_{1}(u)$. From (41) we have for sufficiently large $u$ that

$$
\sup _{t \in[0, T] \backslash E_{4}(u)} \operatorname{Var}\left(\frac{X(t)}{u+c t} m(u)\right) \leq 1-\mathbb{Q}\left(\frac{\ln (m(u))}{m(u)}\right)^{2} .
$$

Further, by BII

$$
\begin{aligned}
(m(u))^{2} \operatorname{Var}\left(\frac{X(t)}{u+c t}-\frac{X(s)}{u+c s}\right) & \leq 2(m(u))^{2}\left(\frac{\sigma^{2}(|t-s|)}{(u+c t)^{2}}+\frac{\sigma^{2}(s) c^{2}(t-s)^{2}}{(u+c t)^{2}(u+c s)^{2}}\right) \\
& \leq \mathbb{Q}\left(\sigma^{2}(|t-s|)+|t-s|^{2}\right) \leq \mathbb{Q}|t-s|^{\alpha_{0}}, \quad s, t \in[0, T] .
\end{aligned}
$$

Consequently, in light of Piterbarg Theorem [Theorem 8.1 in [22]] we have that for $u$ sufficiently large

$$
\lambda_{1}(u) \leq \mathbb{Q}_{1}(m(u))^{2 / \alpha_{0}} \Psi\left(\frac{m(u)}{\sqrt{1-\mathbb{Q}\left(\frac{\ln (m(u))}{m(u)}\right)^{2}}}\right)
$$

Combination of (90), (96) and (97) leads to

$$
\mathbb{P}\left(\frac{1}{\Delta(u)} \int_{0}^{T} \mathbb{I}_{u}(X(t)-c t) \mathrm{d} t>x\right) \sim \mathcal{B}_{B_{2 \alpha_{0}}}(x) \frac{\sigma(T)}{\dot{\sigma}(T)} \frac{1}{(m(u))^{2} \Delta(u)} \Psi(m(u)),
$$

establishing the claim.

Case $\lim _{t \rightarrow 0} \frac{|t|}{\sigma^{2}(|t|)}=1 / \theta \in(0, \infty)$. First note that (91)-(95) still hold. Next we check the conditions of ii) in Theorem 2.1. Following the notation in Theorem 2.1, we have that

$$
\begin{aligned}
& \gamma=\lim _{u \rightarrow \infty} n^{2}(u) w(g(u))=2 \sigma(T) \dot{\sigma}(T) \lim _{u \rightarrow \infty} \frac{\overleftarrow{\sigma}\left(u^{-1}\right)}{\sigma^{2}\left(\overleftarrow{\sigma}\left(u^{-1}\right)\right)}=\frac{2 \sigma(T) \dot{\sigma}(T)}{\theta}, \\
& a_{1}=\lim _{u \rightarrow \infty} a_{1}(u)=0, \quad a_{2}=\lim _{u \rightarrow \infty} a_{2}(u)=\lim _{u \rightarrow \infty}(\Delta(u))^{-1}\left(\frac{\ln m(u)}{m(u)}\right)^{2}=\infty
\end{aligned}
$$

Consequently, in light of case ii) in Theorem 2.1 and Remark 2.2

$$
\lambda(u) \sim \mathcal{B}_{B_{2 \alpha_{0}}}^{\frac{2 \sigma(T) \dot{\sigma}(T)|t|}{\theta}}(x) \Psi(m(u)), \quad u \rightarrow \infty
$$

which together with (90) and (97) establishes the claim.

Case $\lim _{t \rightarrow 0} \frac{|t|}{\sigma^{2}(|t|)}=\infty$. Noting that $v(u)=(m(u))^{2}$, scaling of time by $\Delta(u)$ we have

$$
\lambda(u)=\mathbb{P}\left(\int_{E_{5}(u)} \mathbb{I}_{0}\left(Z_{u}(t)-m(u)\right) \mathrm{d} t>\frac{x}{\Delta(u)(m(u))^{2}}\right),
$$

with $E_{5}(u)$ defined in $(92)$ and

$$
Z_{u}(t)=\frac{X(T-\Delta(u) t)}{u+c T-c \Delta(u) t} m(u)
$$

Next we verify conditions of case iii) in Theorem 2.1. Notice that (91)-(95) still hold. Using notation in Theorem 2.1, we have

Moreover,

$$
\begin{gathered}
\gamma=\lim _{u \rightarrow \infty} n^{2}(u) w(g(u))=2 \sigma(T) \dot{\sigma}(T) \lim _{u \rightarrow \infty} \frac{\overleftarrow{\sigma}\left(u^{-1}\right)}{\sigma^{2}\left(\overleftarrow{\sigma}\left(u^{-1}\right)\right)}=\infty \\
b_{1}=\lim _{u \rightarrow \infty} \frac{a_{1}(u) g(u)}{\overleftarrow{w}\left(n^{-2}(u)\right)}=0, b_{2}=\lim _{u \rightarrow \infty} \frac{b(u) g(u)}{\overleftarrow{w}\left(n^{-2}(u)\right)}=\lim _{u \rightarrow \infty} \frac{\dot{\sigma}(T)}{\sigma(T)} \frac{(\ln m(u))^{2}}{m(u)}=\infty
\end{gathered}
$$

$$
\frac{x}{\Delta(u)(m(u))^{2}}=\frac{\overleftarrow{w}\left(n^{-2}(u)\right)}{g(u)} \frac{\dot{\sigma}(T)}{\sigma(T)} x=\theta(u) \frac{\dot{\sigma}(T)}{\sigma(T)} x
$$

Consequently, by case iii) in Theorem 2.1 and Remark 2.2

$$
\lambda(u)=\mathbb{P}\left(\int_{E_{5}(u)} \mathbb{I}_{0}\left(Z_{u}(t)-m(u)\right) \mathrm{d} t>\frac{\overleftarrow{w}\left(n^{-2}(u)\right)}{g(u)} \frac{\dot{\sigma}(T)}{\sigma(T)} x\right) \sim \mathcal{B}_{0}^{|t|}\left(\frac{\dot{\sigma}(T)}{\sigma(T)} x,[0, \infty)\right) \Psi(m(u)), \quad u \rightarrow \infty
$$


which combined with (90), (97) and (19) yields that

$$
\mathbb{P}\left((m(u))^{2} \int_{0}^{T} \mathbb{I}_{u}(X(t)-c t) \mathrm{d} t>x\right) \sim e^{-\frac{\dot{\sigma}(T)}{\sigma(T)} x} \Psi(m(u)), \quad u \rightarrow \infty .
$$

This completes the proof.

Proof of Corollary 3.5. Observe that for any $y \geq 0$

$$
\mathbb{P}\left(\frac{\dot{\sigma}(T)}{\sigma^{3}(T)} u^{2}\left(T-\tau_{u, T}^{*}\left(x_{1}, x_{2}\right)\right) \geq y\right)=\frac{\mathbb{P}\left(\tau_{u, T}\left(x_{2}\right) \leq T_{u}(y)\right)}{\mathbb{P}\left(\tau_{u, T}\left(x_{1}\right) \leq T\right)},
$$

with $T_{u}(y)=T-u^{-2} \frac{\sigma^{3}(T)}{\dot{\sigma}(T)} y$. By the definition of $\tau_{u, T}(x)$, we have

$$
\begin{aligned}
\mathbb{P}\left(\tau_{u, T}\left(x_{1}\right) \leq T\right) & =\mathbb{P}\left(v(u) \int_{0}^{T} \mathbb{I}_{u}(X(t)-c t) \mathrm{d} t>x_{1}\right), \\
\mathbb{P}\left(\tau_{u, T}\left(x_{2}\right) \leq T_{u}(y)\right) & =\mathbb{P}\left(v(u) \int_{0}^{T_{u}(y)} \mathbb{I}_{u}(X(t)-c t) \mathrm{d} t>x_{2}\right),
\end{aligned}
$$

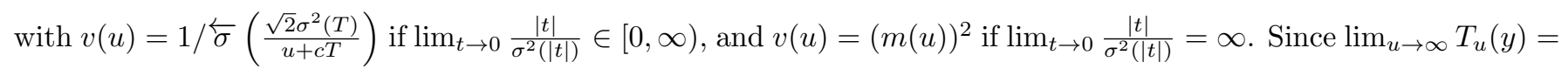
$T$, we get that the asymptotics of (99) is the same as in Theorem 3.4 with $T$ replaced by $T_{u}(y)$ and $x$ replaced by $x_{2}$. Using this fact and by Theorem 3.4 for all $\lim _{t \rightarrow 0} \frac{|t|}{\sigma^{2}(|t|)} \in[0, \infty]$

$$
\begin{aligned}
\frac{\mathbb{P}\left(\tau_{u, T}\left(x_{2}\right) \leq T_{u}(y)\right)}{\mathbb{P}\left(\tau_{u, T}\left(x_{1}\right) \leq T\right)} & \sim \Gamma\left(x_{1}, x_{2}\right) \frac{\Psi\left(\frac{u+c T_{u}(y)}{\sigma\left(T_{u}(y)\right)}\right)}{\Psi(m(u))} \\
& \sim \Gamma\left(x_{1}, x_{2}\right) \exp \left(-\frac{1}{2}\left(\frac{u+c T}{\sigma(T)}\right)^{2}\left(\left(1-\frac{c\left(T-T_{u}(y)\right)}{u+c T}\right)^{2}\left(1-\frac{\dot{\sigma}(T)\left(T-T_{u}(y)\right)}{\sigma(T)}\right)^{-2}-1\right)\right) \\
& \sim \Gamma\left(x_{1}, x_{2}\right) e^{-y}, \quad y \geq 0,
\end{aligned}
$$

where $\Gamma\left(x_{1}, x_{2}\right)$ is given in Theorem 3.5. This completes the proof.

Proof of Theorem 3.6 By self-similarity of $X$, we have that

$$
\begin{aligned}
\mathbb{P}\left(\frac{1}{u \overleftarrow{\rho}\left(\left(\widehat{A} u^{1-H}\right)^{-2}\right)} \int_{0}^{\infty} \mathbb{I}_{u}(X(t)-c t) d t>x\right) & =\mathbb{P}\left(\frac{1}{\overleftarrow{\rho}\left(\left(\widehat{A} u^{1-H}\right)^{-2}\right)} \int_{0}^{\infty} \mathbb{I}_{0}\left(\frac{X(t)}{1+c t}-u^{1-H}\right) d t>x\right) \\
& =\mathbb{P}\left(\frac{1}{\overleftarrow{\rho}\left(\left(\widehat{A} u^{1-H}\right)^{-2}\right)} \int_{0}^{\infty} \mathbb{I}_{0}\left(\widehat{A} \frac{X(t)}{1+c t}-\widehat{A} u^{1-H}\right) d t>x\right)
\end{aligned}
$$

Let

$$
Z(t)=\widehat{A} \frac{X(t)}{1+c t}, \quad n(u)=\widehat{A} u^{1-H}, \quad \Delta(u)=\overleftarrow{\rho}\left((n(u))^{-2}\right)
$$

Observe that

$$
\varpi(u) \leq \mathbb{P}\left(\frac{1}{\Delta(u)} \int_{0}^{\infty} \mathbb{I}_{0}(Z(t)-n(u)) d t>x\right) \leq \varpi(u)+\varpi_{1}(u),
$$

where

$$
\begin{aligned}
\varpi(u) & =\mathbb{P}\left(\frac{1}{\Delta(u)} \int_{\left[t_{0}-(\ln n(u)) / n(u), t_{0}+(\ln n(u)) / n(u)\right]} \mathbb{I}_{u}(Z(t)-n(u)) d t>x\right), \\
\varpi_{1}(u) & =\mathbb{P}\left(\sup _{t \in[0, \infty) \backslash\left[t_{0}-(\ln n(u)) / n(u), t_{0}+(\ln n(u)) / n(u)\right]} Z(t)>n(u)\right) .
\end{aligned}
$$

First we derive the asymptotics of $\varpi(u)$ by applying Theorem 2.1 and then show that $\omega_{1}$ is asymptotically negligible in comparison to $\varpi(u)$ as $u \rightarrow \infty$. We distinguish between three cases: $\gamma=0, \gamma \in(0, \infty)$ and $\gamma=\infty$. 
Case $\gamma=0$.

The asymptotics of $\varpi(u)$. In order to apply Theorem 2.1, we rewrite

$$
\varpi(u)=\mathbb{P}\left(\int_{\left[-\delta_{u}, \delta_{u}\right]} \mathbb{I}_{0}\left(Z\left(t_{0}+\Delta(u) t\right)-n(u)\right) d t>x\right),
$$

with $\delta_{u}=\frac{\ln n(u)}{n(u) \Delta(u)}$. By (30) and (31), we have that, as $u \rightarrow \infty$

$$
1-\sqrt{\operatorname{Var}\left(Z\left(t_{0}+\Delta(u) t\right)\right)} \sim \frac{\widehat{B}}{2 \widehat{A}}(\Delta(u) t)^{2}, \quad t \in\left[-\delta_{u}, \delta_{u}\right],
$$

and

$$
\lim _{u \rightarrow \infty} \sup _{s \neq t, s, t \in\left[-\delta_{u}, \delta_{u}\right]}\left|\frac{n^{2}(u)\left(1-\operatorname{Corr}\left(Z\left(t_{0}+\Delta(u) s\right), Z\left(t_{0}+\Delta(u) t\right)\right)\right)}{\frac{\rho(\Delta(u)|t-s|)}{\rho(\Delta(u))}}-1\right|=0 .
$$

Thus we have that, corresponding to the notation in Theorem 2.1,

$$
w(t)=\frac{\widehat{B}}{2 \widehat{A}} t^{2}, \quad g(u)=\Delta(u), \quad a_{1}(u)=-\delta_{u}, \quad a_{2}(u)=\delta_{u} .
$$

A direct calculation shows that

$$
\begin{gathered}
\lim _{u \rightarrow \infty} n^{2}(u) w(g(u))=\frac{\widehat{B}}{2 \widehat{A}} \lim _{u \rightarrow \infty}(n(u) \Delta(u))^{2}=\frac{\widehat{B}}{2 \widehat{A}} \lim _{u \rightarrow \infty}\left(n(u) \overleftarrow{\rho}\left((n(u))^{-2}\right)\right)^{2}=\frac{\widehat{B}}{2 \widehat{A}} \lim _{t \rightarrow 0} \frac{t^{2}}{\rho(|t|)}=0 \\
\lim _{u \rightarrow \infty} n(u) w\left(g(u)\left|a_{i}(u)\right|\right)=\frac{\widehat{B}}{2 \widehat{A}} \lim _{u \rightarrow \infty} \frac{(\ln n(u))^{2}}{n(u)}=0 \\
x_{i}=\lim _{u \rightarrow \infty} n^{2}(u) w\left(g(u)\left|a_{i}(u)\right|\right)=\frac{\widehat{B}}{2 \widehat{A}} \lim _{u \rightarrow \infty}(\ln n(u))^{2}=\infty, \quad i=1,2
\end{gathered}
$$

By the fact that

$$
\lim _{u \rightarrow \infty} a_{1}(u)=-\lim _{u \rightarrow \infty} \frac{\ln n(u)}{n(u) \Delta(u)}=-\infty, \quad \lim _{u \rightarrow \infty} a_{2}(u)=\infty
$$

we have that

$$
y_{1}=-\infty, \quad y_{2}=\infty \text {. }
$$

Noting that $\lim _{u \rightarrow \infty} \Delta(u)=0$ and by i) in Theorem 2.1 and Remark $2.2 \mathrm{i}$ ), we have

$$
\begin{aligned}
\varpi(u) & \sim \mathcal{B}_{B_{\alpha}}(x) 2^{-1} \int_{-\infty}^{\infty}|t|^{-1 / 2} e^{-|t|} d t \frac{\sqrt{2 \widehat{A} / \widehat{B}}}{n(u) \Delta(u)} \Psi(n(u)) \\
& \sim \mathcal{B}_{B_{\alpha}}(x) \sqrt{\frac{2 \widehat{A} \pi}{\widehat{B}}} \frac{1}{\overleftarrow{\rho}\left(\left(\widehat{A} u^{1-H}\right)^{-2}\right) \widehat{A} u^{1-H}} \Psi\left(\widehat{A} u^{1-H}\right)
\end{aligned}
$$

Upper bound of $\varpi_{1}(u)$. Observe that, for $u$ sufficiently large,

$$
\varpi_{1}(u) \leq \sum_{i=2}^{4} \varpi_{i}(u)
$$

where

$$
\begin{gathered}
\varpi_{2}(u)=\mathbb{P}\left(\sup _{t \in[0, \epsilon]} Z(t)>n(u)\right), \quad \varpi_{3}(u)=\mathbb{P}\left(\sup _{\left.t \in[\epsilon, L] \backslash t_{0}-(\ln n(u)) / n(u), t_{0}+(\ln n(u)) / n(u)\right]} Z(t)>n(u)\right), \\
\varpi_{4}(u)=\sum_{k=0}^{\infty} \mathbb{P}\left(\sup _{t \in[L+k, L+k+1]} Z(t)>n(u)\right),
\end{gathered}
$$

with $L>t_{0}$. In order to prove that $\varpi_{1}(u)=o(\varpi(u)), u \rightarrow \infty$, it suffices to show that $\varpi_{i}(u), i=2,3,4$ are negligible compared with $\varpi(u)$ respectively. We begin with $\varpi_{2}(u)$. Using the fact that for $\epsilon>0$ sufficiently small there exists $0<\delta<1$ such that

$$
\sup _{t \in[0, \epsilon]} \operatorname{Var}(Z(t))<1-\delta
$$


and by Borell-TIS inequality (see [31, 32]), we have

$$
\varpi_{2}(u) \leq e^{-\frac{\left(n(u)-\mathbb{E}\left\{\sup _{t \in[0, \epsilon]} Z(t)\right\}\right)^{2}}{2(1-\delta)}}=o(\varpi(u)), \quad u \rightarrow \infty .
$$

Next we focus on $\varpi_{3}(u)$. By $(30)$ and self-similarity of $X$, we have that for $s, t \geq \epsilon>0$ and $r=\frac{t_{0}}{t}$

$$
\begin{aligned}
\mathbb{E}\left\{(\bar{X}(t)-\bar{X}(s))^{2}\right\} & =2(1-\operatorname{Corr}(X(s), X(t))) \\
& =2(1-\operatorname{Corr}(X(r s), X(r t))) \\
& \leq 4 \rho(r|s-t|) \leq 8\left(t_{0} / \epsilon\right)^{\alpha / 2}|t-s|^{\alpha / 2}, \quad|s-t| \rightarrow 0,
\end{aligned}
$$

which indicates that for $s, t \geq \epsilon>0$ and $|s-t| \leq L_{1}<\infty$ with $L_{1}$ a positive constant

$$
\mathbb{E}\left\{(\bar{X}(t)-\bar{X}(s))^{2}\right\} \leq \mathbb{Q}|t-s|^{\alpha / 2}
$$

Hence (31) and Piterbarg inequality (Lemma 8.1 in [22]) leads to

$$
\begin{aligned}
\varpi_{3}(u) & \leq \mathbb{P}\left(\sup _{t \in\left[\epsilon, 2 t_{0}\right]} \bar{X}(t)>\frac{n(u)}{1-\mathbb{Q}_{1}(\ln n(u))^{2} / n^{2}(u)}\right) \\
& \leq \mathbb{Q}(n(u))^{4 / \alpha} \Psi\left(\frac{n(u)}{1-\mathbb{Q}_{1}(\ln n(u))^{2} / n^{2}(u)}\right) \\
& =o(\varpi(u)), \quad u \rightarrow \infty .
\end{aligned}
$$

Finally, we consider $\varpi_{4}(u)$. Using the fact that

$$
\sup _{t \in[L+k, L+k+1]} \operatorname{Var}(Z(t)) \leq\left(\frac{\widehat{A}}{c}(L+k)^{H-1}\right)^{2}
$$

and (106), by Piterbarg inequality we have that, for $L$ sufficiently large,

$$
\begin{aligned}
\varpi_{4}(u) & \leq \sum_{k=0}^{\infty} \mathbb{P}\left(\sup _{t \in[L+k, L+k+1]} \bar{X}(t)>\frac{n(u)}{\frac{\widehat{A}}{c}(L+k)^{H-1}}\right) \\
& \leq \sum_{k=0}^{\infty} \mathbb{Q}(n(u))^{4 / \alpha} \Psi\left(\frac{n(u)}{\frac{\widehat{A}}{c}(L+k)^{H-1}}\right) \\
& \leq \mathbb{Q}(n(u))^{4 / \alpha} \Psi\left(\frac{n(u)}{\mathbb{Q}_{1} L^{H-1}}\right)=o(\varpi(u)), \quad u \rightarrow \infty
\end{aligned}
$$

Consequently, we conclude that

$$
\varpi_{1}(u)=o(\varpi(u)), \quad u \rightarrow \infty
$$

Therefore,

$$
\mathbb{P}\left(\frac{1}{u \overleftarrow{\rho}\left(\left(\widehat{A} u^{1-H}\right)^{-2}\right)} \int_{0}^{\infty} \mathbb{I}_{u}(X(t)-c t) \mathrm{d} t>x\right) \sim \mathcal{B}_{B_{\alpha}}(x) \sqrt{\frac{2 \widehat{A} \pi}{\widehat{B}}} \frac{1}{\overleftarrow{\rho}\left(\left(\widehat{A} u^{1-H}\right)^{-2}\right) \widehat{A} u^{1-H}} \Psi\left(\widehat{A} u^{1-H}\right)
$$

This establishes the claim for $\gamma=0$.

Case $\gamma \in(0, \infty)$.

The asymptotics of $\varpi(u)$. Note that (102)-(104) also hold for $\gamma \in(0, \infty)$. As in (105), we have that

$$
\lim _{u \rightarrow \infty} n^{2}(u) w(g(u))=\frac{\widehat{B}}{2 \widehat{A}} \lim _{t \rightarrow 0} \frac{t^{2}}{\rho(|t|)}=\frac{\widehat{B}}{2 \widehat{A}} \gamma \in(0, \infty) .
$$

Moreover,

$$
\lim _{u \rightarrow \infty} g(u) a_{i}(u)=0, \quad \lim _{u \rightarrow \infty} a_{1}(u)=-\lim _{u \rightarrow \infty} \frac{\ln n(u)}{n(u) \Delta(u)}=-\infty, \quad \lim _{u \rightarrow \infty} a_{2}(u)=\infty .
$$

Hence by ii) in Theorem 2.1, we have that

$$
\varpi(u) \sim \widehat{\mathcal{B}}_{B_{2}}^{\frac{\widehat{B} \gamma t^{2}}{2 \hat{A}}}(x) \Psi\left(\widehat{A} u^{1-H}\right)
$$


which combined with (107) and (20) establishes the claim.

Case $\gamma=\infty$.

The asymptotics of $\varpi(u)$. Using the notation for $Z(t), \Delta(u), n(u), \delta_{u}, \varpi_{1}(u)$ in (100)-(102), we have that

$$
\varpi_{5}(u) \leq \mathbb{P}\left(\int_{0}^{\infty} \mathbb{I}_{u}(X(t)-c t) d t>x u^{H}\right) \leq \varpi_{1}(u)+\varpi_{5}(u),
$$

where

$$
\varpi_{5}(u)=\mathbb{P}\left(\int_{\left[-\delta_{u}, \delta_{u}\right]} \mathbb{I}_{0}\left(Z\left(t_{0}+\Delta(u) t\right)-n(u)\right) d t>\frac{x u^{H-1}}{\Delta(u)}\right) .
$$

We focus on $\varpi_{5}(u)$. Note that in this case, (103)-(104) still hold. Following the notation in Theorem 2.1, we have that

$$
\lim _{u \rightarrow \infty} n^{2}(u) w(g(u))=\frac{\widehat{B}}{2 \widehat{A}} \lim _{t \rightarrow 0} \frac{t^{2}}{\rho(|t|)}=\infty, \quad \lim _{u \rightarrow \infty} g(u)\left|a_{i}(u)\right|=\lim _{u \rightarrow \infty} \frac{\ln n(u)}{n(u)}=0, i=1,2 .
$$

Moreover,

$$
b_{1}=\lim _{u \rightarrow \infty} \frac{a_{1}(u) g(u)}{\overleftarrow{w}\left(n^{-2}(u)\right)}=-\sqrt{\frac{\widehat{B}}{2 \widehat{A}}} \lim _{u \rightarrow \infty} \ln n(u)=-\infty, \quad b_{2}=\lim _{u \rightarrow \infty} \frac{a_{2}(u) g(u)}{\overleftarrow{w}\left(n^{-2}(u)\right)}=\sqrt{\frac{\widehat{B}}{2 \widehat{A}}} \lim _{u \rightarrow \infty} \ln n(u)=\infty .
$$

In this case, using the notation in (104) we have

$$
\frac{x u^{H-1}}{\Delta(u)}=x \sqrt{\frac{\widehat{A} \widehat{B}}{2}} \overleftarrow{w}\left(n^{-2}(u)\right) / g(u)
$$

In light of iii) in Theorem 2.1, we have that

$$
\begin{aligned}
\varpi_{5}(u) & =\mathbb{P}\left(\int_{\left[-\delta_{u}, \delta_{u}\right]} \mathbb{I}_{0}\left(Z\left(t_{0}+\Delta(u) t\right)-n(u)\right) d t>x \sqrt{\frac{\widehat{A} \widehat{B}}{2}} \overleftarrow{w}\left(n^{-2}(u)\right) / g(u)\right) \\
& \sim \mathcal{B}_{0}^{t^{2}}\left(\sqrt{\frac{\widehat{A} \widehat{B}}{2}} x,(-\infty, \infty)\right) \Psi(n(u))
\end{aligned}
$$

which together with (107) and (19) completes the proof.

Proof of Theorem 3.7 It follows that

$$
\mathbb{P}\left(v(u) \int_{0}^{T} \mathbb{I}_{u}(X(t)-c t) \mathrm{d} t>x\right)=\mathbb{P}\left(v(u) \int_{0}^{T} \mathbb{I}_{0}\left(\frac{X(t)}{u+c t} \frac{u+c T}{T^{2 H}}-\frac{u+c T}{T^{2 H}}\right) \mathrm{d} t>x\right),
$$

where

$$
X_{u}(t)=\frac{X(t)}{u+c t} \frac{u+c T}{T^{2 H}}
$$

Direct calculation shows that

$$
\lim _{u \rightarrow \infty} \lim _{t \rightarrow T, t<T}\left|\frac{1-\operatorname{Var}\left(X_{u}(t)\right)}{|T-t|}-\frac{H}{T}\right|=0
$$

Moreover, by $\mathbf{S}$ with $t_{0}=T$,

$$
\lim _{\epsilon \rightarrow 0} \sup _{s \neq t, T-\epsilon \leq s, t \leq T}\left|\frac{1-\operatorname{Corr}\left(X_{u}(t), X_{u}(s)\right)}{\rho(|t-s|)}-1\right|=0 .
$$

The local behavior of variance and correlation functions is the same as the one in Theorem 3.4 (see Lemma 4.4). Hence proceeding similarly as in the proof of Theorem 3.4, that is replacing $\frac{\dot{\sigma}(T)}{\sigma(T)}$ by $\frac{H}{T}, \frac{\sigma^{2}(\cdot)}{2 \sigma^{2}(T)}$ by $\rho(\cdot)$ and $\sigma(T)$ by $T^{H}$ in the proof of Theorem 3.4, we establish the claim. 


\section{Appendix}

Proof of (48). In order to prove (48), it suffices to prove that for $c_{1}>0$

$$
\int_{0}^{g(u) a_{2}(u)} e^{-c_{1} n^{2}(u) w(t)} \mathrm{d} t \sim c_{1}^{-1 / \beta} \overleftarrow{w}\left(n^{-2}(u)\right) \beta^{-1} \int_{0}^{c_{1} y_{2}} t^{1 / \beta-1} e^{-t} \mathrm{~d} t, \quad u \rightarrow \infty
$$

Recall that $w$ is a regularly varying function at 0 with index $\beta>0$ satisfying

$$
\lim _{u \rightarrow \infty} n^{2}(u) w\left(g(u) a_{2}(u)\right)=y_{2}>0
$$

where $a_{2}(u)>0$ and $\lim _{u \rightarrow \infty} g(u)=\infty$ and $\lim _{u \rightarrow \infty} g(u) a_{2}(u)=0$. We can assume that $w(x)=\ell(x) x^{\beta}$ with $\ell$ normalized slowly varying function at 0 . Then from [24], we know that $\ell(x) x^{\beta}$ is ultimately monotone for any $\beta \neq 0$, $\ell$ is continuously differentiable and

$$
\lim _{x \rightarrow 0} \frac{x \ell^{\prime}(x)}{\ell(x)}=0
$$

Let $c_{1} n^{2}(u) w(t)=s$. Then we have that

$$
\int_{0}^{g(u) a_{2}(u)} e^{-c_{1} n^{2}(u) w(t)} \mathrm{d} t=\frac{1}{c_{1} n^{2}(u)} \int_{0}^{c_{1} n^{2}(u) w\left(g(u) a_{2}(u)\right)} \frac{1}{w^{\prime}\left(\overleftarrow{w}\left(\frac{s}{c_{1} n^{2}(u)}\right)\right)} e^{-s} \mathrm{~d} s
$$

By (108), it follows that

$$
w^{\prime}(x) \sim \beta \frac{w(x)}{x}, \quad x \rightarrow 0
$$

Hence

$$
\begin{aligned}
\int_{0}^{g(u) a_{2}(u)} e^{c_{1} n^{2}(u) w(t)} \mathrm{d} t & \sim \int_{0}^{c_{1} n^{2}(u) w\left(g(u) a_{2}(u)\right)} \frac{\overleftarrow{w}\left(\frac{s}{c_{1} n^{2}(u)}\right)}{\beta s} e^{-s} \mathrm{~d} s \\
& =\frac{\overleftarrow{w}\left(\frac{1}{c_{1} n^{2}(u)}\right)}{\beta} \int_{0}^{c_{1} n^{2}(u) w\left(g(u) a_{2}(u)\right)} \frac{\overleftarrow{w}\left(\frac{s}{c_{1} n^{2}(u)}\right)}{\overleftarrow{w}\left(\frac{1}{c_{1} n^{2}(u)}\right)} s^{-1} e^{-s} \mathrm{~d} s
\end{aligned}
$$

Note that $\overleftarrow{w}$ is a regularly varying function at 0 with index $1 / \beta$. Moreover, using Potter's bound for $\overleftarrow{w}$ (see e.g. [24] or [35] or Lemma 6.1 in [34]), we have that for any $\epsilon \in(0, \min (1,1 / \beta))$ and all $u$ large

$$
\frac{\overleftarrow{w}\left(\frac{s}{c_{1} n^{2}(u)}\right)}{\overleftarrow{w}\left(\frac{1}{c_{1} n^{2}(u)}\right)} \leq(1+\epsilon) s^{1 / \beta-\epsilon}, \quad 0<s<c_{1} n^{2}(u) w\left(g(u) a_{2}(u)\right)
$$

Moreover, since $\overleftarrow{w}$ is regularly varying at 0 , then for any $s>0$

$$
\lim _{u \rightarrow \infty} \frac{\overleftarrow{w}\left(\frac{s}{c_{1} n^{2}(u)}\right)}{\overleftarrow{w}\left(\frac{1}{c_{1} n^{2}(u)}\right)}=s^{1 / \beta}
$$

Hence the dominated convergence theorem implies that

$$
\int_{0}^{g(u) a_{2}(u)} e^{-c_{1} n^{2}(u) w(t)} \mathrm{d} t \sim c_{1}^{-1 / \beta} \overleftarrow{w}\left(n^{-2}(u)\right) \beta^{-1} \int_{0}^{c_{1} y_{2}} s^{1 / \beta-1} e^{-s} \mathrm{~d} s
$$

This completes the proof.

Acknowledgement: The authors would like to thank Enkelejd Hashorva for his comments and suggestions that significantly improved the content of this contribution. The authors also would like to thank the referees for their valuable comments and remarks. K. Dębicki was partially supported by NCN Grant No 2015/17/B/ST1/01102 (2016-2019). Financial support from the Swiss National Science Foundation Grant 200021-175752 is also kindly acknowledged. 


\section{REFERENCES}

[1] S. M. Berman, "Sojourns and extremes of stationary processes," Ann. Probab., vol. 10, no. 1, pp. 1-46, 1982.

[2] S. M. Berman, "Sojourns above a high level for a Gaussian process with a point of maximum variance," Comm. Pure Appl. Math., vol. 38, no. 5, pp. 519-528, 1985.

[3] S. M. Berman, "Extreme sojourns of a Gaussian process with a point of maximum variance," Probability Theory and Related Fields, vol. 74, no. 1, pp. 113-124, 1987.

[4] S. M. Berman, Sojourns and Extremes of Stochastic Processes. The Wadsworth \& Brooks/Cole Statistics/Probability Series, Pacific Grove, CA: Wadsworth \& Brooks/Cole Advanced Books \& Software, 1992.

[5] J. Akahori, "Some formulae for a new type of path-dependent option," Ann. Appl. Probab., vol. 5, no. 2, pp. 383$388,1995$.

[6] A. N. Borodin and P. Salminen, Handbook of Brownian motion-facts and formulae. Probability and its Applications, Birkhäuser Verlag, Basel, second ed., 2002.

[7] R. N. Makarov, "Modeling liquidation risk with occupation times," Int. J. Financ. Eng., vol. 3, no. 4, pp. 1650028, $11,2016$.

[8] H. Guérin and J.-F. Renaud, "Joint distribution of a spectrally negative Lévy process and its occupation time, with step option pricing in view," Adv. in Appl. Probab., vol. 48, no. 1, pp. 274-297, 2016.

[9] H. Guérin and J.-F. Renaud, "On the distribution of cumulative Parisian ruin," Insurance Math. Econom., vol. 73, pp. 116-123, 2017.

[10] D. Landriault, B. Li, and A. L. Mohamed, "On occupation times in the red of Lévy risk models," http://arXiv:1903.03721.

[11] Y. Li and X. Zhou, "On pre-exit joint occupation times for spectrally negative Lévy processes," Statist. Probab. Lett., vol. 94, pp. 48-55, 2014.

[12] B. Li and X. Zhou, "The joint Laplace transforms for diffusion occupation times," Adv. in Appl. Probab., vol. 45, no. 4, pp. 1049-1067, 2013.

[13] H. Zhang, "Occupation times, drawdowns, and drawups for one-dimensional regular diffusions," Adv. in Appl. Probab., vol. 47, no. 1, pp. 210-230, 2015.

[14] R. L. Loeffen, J.-F. Renaud, and X. Zhou, "Occupation times of intervals until first passage times for spectrally negative Lévy processes," Stochastic Process. Appl., vol. 124, no. 3, pp. 1408-1435, 2014.

[15] J. Hüsler and V. Piterbarg, "A limit theorem for the time of ruin in a Gaussian ruin problem," Stochastic Process. Appl., vol. 118, no. 11, pp. 2014-2021, 2008.

[16] E. Hashorva and L. Ji, "Approximation of passage times of $\gamma$-reflected processes with FBM input," J. Appl. Probab., vol. 51, no. 3, pp. 713-726, 2014.

[17] J. Hüsler and V. I. Piterbarg, "Extremes of a certain class of Gaussian processes," Stochastic Process. Appl., vol. 83, no. 2, pp. 257-271, 1999.

[18] K. Dȩbicki, "Ruin probability for Gaussian integrated processes," Stochastic Process. Appl., vol. 98, no. 1, pp. 151$174,2002$.

[19] J. Hüsler and V. I. Piterbarg, "On the ruin probability for physical fractional Brownian motion," Stochastic Process. Appl., vol. 113, no. 2, pp. 315-332, 2004.

[20] A. B. Dieker, "Extremes of Gaussian processes over an infinite horizon," Stochastic Process. Appl., vol. 115, no. 2, pp. 207-248, 2005.

[21] K. Dȩbicki, E. Hashorva, and P. Liu, "Uniform tail approximation of homogenous functionals of Gaussian fields," Adv. Applied Probab, vol. 49, no. 4, pp. 1037-1066, 2017.

[22] V. I. Piterbarg, Asymptotic methods in the theory of Gaussian processes and fields, vol. 148 of Translations of Mathematical Monographs. Providence, RI: American Mathematical Society, 1996.

[23] K. Dębicki, Z. Michna, and X. Peng, "Approximation of sojourn times of Gaussian processes," Accepted for publication in Methodology and Computing in Applied Probability, 2018. 
[24] N. H. Bingham, C. M. Goldie, and J. L. Teugels, Regular variation, vol. 27 of Encyclopedia of Mathematics and its Applications. Cambridge University Press, Cambridge, 1989.

[25] K. Dȩbicki and P. Liu, "Extremes of stationary Gaussian storage models," Extremes, vol. 19, no. 2, pp. 273-302, 2016.

[26] K. Dębicki, E. Hashorva, and P. Liu, "Extremes of $\gamma$-reflected Gaussian process with stationary increments," ESAIM Probab. Stat., vol. 21, pp. 495-535, 2017.

[27] Y. Mishura and M. Zili, Stochastic analysis of mixed fractional Gaussian processes. ISTE Press, London; Elsevier Ltd, Oxford, 2018.

[28] T. Bojdecki, L. G. Gorostiza, and A. Talarczyk, "Sub-fractional Brownian motion and its relation to occupation times," Statist. Probab. Lett., vol. 69, no. 4, pp. 405-419, 2004.

[29] M. Zili, "Generalized fractional Brownian motion," Modern Stochastics: Theory and Applications, vol. 4, no. 1, pp. 15-24, 2017.

[30] E. Hashorva and L. Ji, "Piterbarg theorems for chi-processes with trend," Extremes, vol. 18, no. 1, pp. 37-64, 2015.

[31] G. Samorodnitsky, "Probability tails of Gaussian extrema," Stochastic Process. Appl., vol. 38, no. 1, pp. 55-84, 1991.

[32] R. J. Adler and J. E. Taylor, Random fields and geometry. Springer Monographs in Mathematics, New York: Springer, 2007.

[33] K. Dȩbicki and M. Mandjes, Queues and Lévy Fluctuation Theory. Springer International Publishing, 2015.

[34] K. Dȩbicki, E. Hashorva, and P. Liu, "Extremes of Gaussian processes with regularly varying dependence structure," Extremes, vol. 20, no. 2, pp. 333-392, 2017.

[35] P. Soulier, Some applications of regular variation in probability and statistics. Instituto Venezolano de Investigaciones Cientcas: XXII ESCUELA VENEZOLANA DE MATEMATICAS, 2009.

Krzysztof Dȩbicki, Mathematical Institute, University of WrocŁaw, Pl. Grunwaldzki 2/4, 50-384 Wroc£aw, Poland

E-mail address: Krzysztof.Debicki@math.uni.wroc.pl

Peng Liu, Department of Actuarial Science, Faculty of Business and Economics, University of Lausanne, Unil-Dorigny 1015

Lausanne, Switzerland and Department of Statistics and Actuarial Science, University of Waterloo, Canada

E-mail address: peng.liu1@uwaterloo.ca

Zbigniew Michna, Department of Mathematics and Cybernetics, WrocŁaw University of Economics, Poland

E-mail address: zbigniew.michna@ue.wroc.pl 